\title{
Intercomparison of Three Microwave/Infrared High Resolution Line-by-Line Radiative Transfer Codes
}

\author{
Franz Schreier ${ }^{\mathrm{a}, *}$, Mathias Milz ${ }^{\mathrm{b}}$, Stefan A. Buehler ${ }^{\mathrm{c}}$, Thomas von Clarmann $^{\mathrm{d}}$ \\ ${ }^{a}$ DLR - German Aerospace Center, Remote Sensing Technology Institute, \\ 82234 Oberpfaffenhofen, GERMANY \\ ${ }^{b}$ Luleå University of Technology, Department of Computer Science, Electrical and Space Engineering, 98128 Kiruna, swEDEN \\ ${ }^{c}$ Universität Hamburg, Meteorological Institute, Bundesstraße 55, 20146 Hamburg, GERMANY \\ ${ }^{d}$ KIT - Karlsruhe Institute of Technology, Institute of Meteorology and Climate Research, 76344 Leopoldshafen, GERMANY
}

\begin{abstract}
An intercomparison of three line-by-line (lbl) codes developed independently for atmospheric radiative transfer and remote sensing - ARTS, GARLIC, and KOPRA - has been performed for a thermal infrared nadir sounding application assuming a HIRS-like (High resolution Infrared Radiation Sounder) setup. Radiances for the 19 HIRS infrared channels and a set of 42 atmospheric profiles from the "Garand dataset" have been computed.

The mutual differences of the equivalent brightness temperatures are presented and possible causes of disagreement are discussed. In particular, the impact of path integration schemes and atmospheric layer discretization is assessed. When the continuum absorption contribution is ignored because of the different implementations, residuals are generally in the sub-Kelvin range and smaller than $0.1 \mathrm{~K}$ for some window channels (and all atmospheric models and lbl codes). None of the three codes turned out to be perfect for all channels and atmospheres. Remaining discrepancies are attributed to different lbl optimization techniques. Lbl codes seem to have reached a maturity in the implementation of radiative transfer that the choice of the underlying physical models (line shape models, continua etc) becomes increasingly relevant.
\end{abstract}

Keywords: Line-by-line; Infrared; HIRS channels; Garand atmospheres; Intercomparisons

\section{Introduction}

Radiative transfer plays an important role in atmospheric science. For the analysis of an increasing number of high resolution microwave and infrared (IR) spectroscopic observations of Earth's or planetary atmospheres as well as for the generation and verification of low resolution models, line-by-line (lbl) models are indispensable. Clearly, the quality of remote sensing products critically depends on the accuracy of the radiative transfer codes used as a forward model in the inversion process. Accordingly, verification and validation [1] of these codes is crucial, and several code intercomparisons were performed in the past, e.g. Fischer et al. 2], Ellingson and Fouquart [3], Glatthor et al. 4], Soden et al. 5], Garand et al. [6], von Clarmann et al. [7, 8, Tjemkes et al. 9, Kratz et al. 10, Melsheimer et al. [11, Buehler et al. [12, Saunders et al. [13. Further intercomparisons in the context of planetary science and climate modeling were presented by, e.g., Sromovsky et al. [14, Yang et al. [15].

To our knowledge, in most of these studies one of the participating models has been selected as reference code in case difference spectra were shown. This is clearly a

\footnotetext{
* Corresponding author

Email addresses: franz.schreier@dlr.de (Franz Schreier), mathias.milz@ltu.se (Mathias Milz)
}

natural approach when the number of models to be analyzed is "large". For example, five independent radiative transfer codes have been intercompared against the Karlsruhe Optimized \& Precise Radiative transfer Algorithm (KOPRA) 16 in the AMIL2DA (Advanced MIpas Level 2 Data Analysis; MIPAS = Michelson Interferometer for Passive Atmospheric Sounding) forward model intercomparison experiment [7. Likewise, the Atmospheric Radiative Transfer Simulator (ARTS) [17, 18 served as the "truth" in the intercomparison of microwave codes 11 in the context of the "Third International Radiative Transfer Modeling Workshop" (IRTMW3).

Some years later Milz 19 presented a comparison of the ARTS and KOPRA models in the IR spectral range. Here we extend this intercomparison with GARLIC, the Generic Atmospheric Radiation Line-by-line Infrared Code 20, whose Fortran 77 predecessor "MIRART-SQuIRRL" (Modular InfraRed Atmospheric Radiative Transfer Schwarzschild Quadrature IR Radiation Lbl) had been participating in both the AMIL2DA and IRTMW3 studies. First results of the intercomparison have been presented at the International Radiation Symposium 2012 [21].

This paper is organized in five sections: In the next section, we introduce the essentials of infrared radiative transfer with high spectral resolution (i.e., lbl modeling) and continue with a brief review of the three codes con- 
sidered here. The setup of the intercomparison study is described in Section 3 , and the results are presented in Section 4. A summary and conclusions are given in Section 5 .

\section{Theory and Methods}

\subsection{Atmospheric infrared radiative transfer}

In a gaseous, non-scattering atmosphere in local thermodynamic equilibrium radiative transfer [22] is described by the Schwarzschild equation 23-25, and the intensity (radiance) $I$ at wavenumber $\nu$ is given by the integral along the line-of-sight

$$
I(\nu)=I_{\mathrm{b}}(\nu) \mathrm{e}^{-\tau_{\mathrm{b}}(\nu)}+\int_{0}^{\tau_{\mathrm{b}}(\nu)} B\left(\nu, T\left(\tau^{\prime}\right)\right) \mathrm{e}^{-\tau^{\prime}} \mathrm{d} \tau^{\prime}
$$

where $B$ denotes Planck's function for a black-body with temperature $T$ and $I_{\mathrm{b}}(\nu)$ describes a background contribution (e.g., due to the surface in case of a nadir-viewing observer). The optical depth $\tau$ (measured relative to the observer at position $s=0$, equivalent to $\tau=0$ ) is closely related to the monochromatic transmission

$$
\begin{aligned}
\mathcal{T}(\nu, s) & =\mathrm{e}^{-\tau(\nu, s)} \\
& =\exp \left(-\int_{0}^{s} \mathrm{~d} s^{\prime} \sum_{m} k_{m}\left(\nu, p\left(s^{\prime}\right), T\left(s^{\prime}\right)\right) n_{m}\left(s^{\prime}\right)\right),
\end{aligned}
$$

where $p$ is the atmospheric pressure and the integrand constitutes the absorption coefficient, essentially determined by the sum of the absorption cross sections $k_{m}$ scaled by the molecular number densities $n_{m}$. Note that the contribution of the pressure and temperature dependent continuum absorption 26], slowly varying with wavenumber, has not been included in Eq. (2).

In high resolution lbl models, the absorption cross section of molecule $m$ is given by the superposition of many lines $l$ with line center positions $\hat{\nu}_{l}$, each described by the product of a temperature-dependent line strength $S_{l}$ and a normalized line shape function $g$ describing the broadening mechanism(s) (for brevity the subscript $m$ is omitted),

$$
k(\nu, p, T)=\sum_{l} S_{l}(T) g\left(\nu ; \hat{\nu}_{l}, \gamma_{l}(p, T)\right) .
$$

For the infrared and microwave spectral regime, the combined effect of pressure broadening (corresponding to a Lorentzian line shape $g_{\mathrm{L}}$ ) and Doppler broadening (corresponding to a Gaussian line shape $g_{\mathrm{G}}$ ) can be represented by a convolution of both, i.e. the Voigt line profile 27 .

$$
g_{\mathrm{V}}\left(\nu-\hat{\nu}, \gamma_{\mathrm{L}}, \gamma_{\mathrm{G}}\right)=g_{\mathrm{L}}\left(\nu-\hat{\nu}, \gamma_{\mathrm{L}}\right) \otimes g_{\mathrm{G}}\left(\nu-\hat{\nu}, \gamma_{\mathrm{G}}\right)
$$

where the Lorentz width $\gamma_{\mathrm{L}}$ is depending on pressure and temperature and the Gaussian width $\gamma_{\mathrm{G}}$ depends on temperature and the molecular mass. It should be noted that the increasing quality of ground-based and, more recently, space-based spectrometers has indicated the approximative nature of the Voigt (and Lorentz) profile, i.e. effects due to Dicke narrowing, speed-dependent broadening, or line mixing have to be taken into account by more sophisticated line profiles [e.g. 28, 29] and a corresponding extension of the line parameter databases [e.g. 30].

Instrumental effects are modeled by convolution of the monochromatic intensity (1) or transmission (2) with appropriate spectral response functions $(\mathrm{SRF}) \mathcal{S}(\nu)$. The equivalent brightness temperatures shown below are computed from the convolved radiances $\hat{I}$ using the inverse of Planck's function $T_{\mathrm{B}} \equiv B^{-1}(\nu, \hat{I})$ with the wavenumber given by the (tabulated) SRF center position. Furthermore, the effect of the finite aperture can be simulated by numerical integration of the radiances over the finite instantaneous field of view.

\subsection{ARTS - Atmospheric Radiative Transfer Simulator}

ARTS is a publicly available radiative transfer code published under the GNU license agreement (see also http: //www.radiativetransfer.org/). It is an open-source project driven by the University of Hamburg, Germany and Chalmers University, Gothenburg, Sweden [17, 18, 31]. ARTS was originally developed for applications in the microwave range but is also suitable for applications in the mid- and far-infrared range [see e.g. 32, 33]. In this study we used the stable version 2.2 [18]. ARTS was included in intercomparisons for microwave radiative transfer (up, down, and limb) [11] as well as infrared radiative transfer models simulating AIRS (Atmospheric Infrared Sounder) radiances 13 . It is able to treat clear sky conditions with $\mathrm{lbl}$ and continuum absorption as well as different scattering schemes for hydrometeors. For this study, the MlawerTobin-Clough-Kneizys-Davies (MT-CKD) 1.0 continua for $\mathrm{O}_{2}, \mathrm{~N}_{2}, \mathrm{H}_{2} \mathrm{O}$, and $\mathrm{CO}_{2}$ have been used [34, 35 .

Absorption in ARTS is level based. For efficiency, the lbl absorption can be stored in a frequency, pressure, temperature, and water vapor dependent lookup table and reused for many subsequent radiative transfer simulations 36. Besides, for instrument simulation as in this study, ARTS can also be used to compute atmospheric radiative energy fluxes and heating rates [37, 38].

\subsection{GARLIC - Generic Atmospheric Radiation Line-by- line Infrared Code}

GARLIC [20, 39] has been developed for high resolution infrared-microwave atmospheric radiative transfer modeling with a modular approach appropriate for simulation and retrieval in Earth [40, 41] and planetary science 42]. Unlike ARTS, GARLIC is not open source, however, Py4CAtS - Python for Computational Atmospheric Spectroscopy [43] a lightweight implementation of GARLIC, is publically available at https://atmos.eoc.dlr. de/tools/Py4CAtS/. 
In addition to the lbl absorption, the "CKD" continua 34 and collision-induced absorption (CIA) 44 are implemented. For the computation of the Voigt function GARLIC uses a combination of the Humlíček [45] and the Weideman [46] rational approximations [47. For further speed-up of the lbl calculation a multigrid algorithm is used [48. In contrast to most other lbl codes treating the inhomogeneous atmosphere in a layer-by-layer approach, GARLIC is level-oriented and employs numerical quadrature techniques to solve the path integrals. More specifically, the Planck function is assumed to vary either linearly or exponentially with optical depth between two adjacent levels and the Schwarzschild integral (1) is approximated by the trapezoid rule (the linear in $\tau$ mode was termed "trapezoid-Laguerre quadrature" in 20]). Note that in contrast to KOPRA and ARTS no intermediate levels are introduced for the path integration along the line-of-sight.

\subsection{KOPRA - Karlsruhe Optimized \& Precise Radiative transfer Algorithm}

KOPRA [16] is a line-by-line, layer-by-layer model for forward calculation of infrared atmospheric transmittance and radiance spectra for various geometries and was initially developed for the analysis of MIPAS mid infrared limb emission sounder data [49. It is also applicable to upward and nadir looking instruments using thermal emission as well as solar and lunar absorption modes for high resolution spectroscopic and radiometer applications with dedicated spectral response functions. Furthermore, nonLTE radiative transfer is supported [cf. 8, 50. KOPRA uses a layering scheme, and within each layer a CurtisGodson weighting scheme is applied [51, 52].

For this study we used KOPRA version 4.1 .54 with HITRAN (HIgh resolution atmospheric TRANsmission) 2004 spectroscopic data. Like ARTS, the evaluation of the Voigt line shape uses a computationally efficient algorithm by Kuntz [53] of the Humlíček 45] complex error function algorithm. Continuum absorption for $\mathrm{H}_{2} \mathrm{O}$ was based on the CKD 2.4 continuum. The continuum-like far-wing signal of $\mathrm{CO}_{2}$ is using pretabulated absorption cross sections where the relevant chi-factors are considered. $\mathrm{N}_{2}$ and $\mathrm{O}_{2}$ continua follow the models by Lafferty et al. 54 and Thibault et al. 55], respectively. [For more information see 56.

\section{Setup}

In this intercomparison we consider a thermal infrared nadir sounding application and model the upwelling radiation seen by a spaceborne downlooking observer (viewing angle $180^{\circ}$ from zenith). In particular we use a HIRS (High resolution Infrared Radiation Sounder) setup and compute radiances for the 19 HIRS infrared channels of NOAA 17. Fig. 1 shows the upwelling radiation as seen by an observer form space assuming a US Standard atmosphere [58. Furthermore, the middle and bottom plots indicate the total transmission and the individual molecular contributions. Channels 1 to 7 and 13 to 16 are located in the $\mathrm{CO}_{2} \nu_{2}$ and $\nu_{3}$ bands relevant for atmospheric temperature sounding, whereas channels 8 and 10 and 17 to 19 are in atmospheric window regions and hence should be sensitive to the lowest atmospheric layers. Note that the equivalent brightness temperatures shown in Section 4 correspond to the high resolution radiances convolved with the HIRS response functions; the convolution of brightness temperatures with the response functions would lead to significantly different results.

Radiance spectra were calculated for a set of 42 atmospheric profiles (the Garand et al. 6] dataset, comprising pressure, temperature, and molecular concentrations, Fig. 2) representative of most meteorological cloud-free situations: The first six atmospheres correspond to the AFGL (Air Force Geophysics Laboratory) atmospheres [58, atmospheres $7-18,19-30$, and $31-42$ are ranked by increasing mean temperature, water content, and total ozone, respectively (see also Tab. 2). The data are given on 43 levels with a common pressure grid in the range 0.1 to $1013.25 \mathrm{mb}$ and slightly varying altitudes up to about 61 to $67 \mathrm{~km}$. Note that for two third of the atmospheres the maximum temperature is at the bottom of the atmosphere and for two atmospheres (\# 7 and 31) the maximum temperature exceeds the surface temperature by more than $30 \mathrm{~K}$.

Absorption of the main molecular absorbers in the infrared is considered $\left(\mathrm{H}_{2} \mathrm{O}, \mathrm{CO}_{2}, \mathrm{O}_{3}, \mathrm{CH}_{4}, \mathrm{~N}_{2} \mathrm{O}, \mathrm{CO}\right.$, $\mathrm{O}_{2}$, and $\mathrm{N}_{2}$, compare Fig. 1 data (transition wavenumber, strength, broadening parameters) taken from the HITRAN 2004 database [59]. Continuum contributions, namely the CKD or MT-CKD continuum, were also taken into account for water vapor, carbon dioxide, nitrogen, and oxygen.

\section{Results}

In the following we present our results essentially in the chronological order as the experiments have been conducted. Recalling the AMIL2DA [7] and IRTMW3 [1] intercomparisons we note that a series of increasingly more complex exercises or cases had been defined by the KOPRA and ARTS teams, respectively, whereas here we started with a single task, i.e. brightness temperature for the 19 HIRS channels and 42 Garand atmospheres. Furthermore, note that all runs have been performed using the codes as is, i.e., only input data and job configurations have been changed. In particular, we have not implemented any changes in the codes.

A distinct feature of most lbl codes is their approach to tackle the computational challenges of line-by-line modelling. Whereas GARLIC and KOPRA use highly optimized Voigt function algorithms [47, 53, 60] along with multigrid techniques, ARTS relies on lookup tables [36]. Furthermore, the most time consuming code segments 
Table 1: Statistics of the Garand atmospheres. $T_{0}$ denotes the temperature at altitude $z=0, \Delta T=T_{\max }-T_{\min }$, and columns $\# 6-8$ characterize the level-to-level temperature difference $\delta T$. Emphasized numbers indicate extreme values.

\begin{tabular}{|c|c|c|c|c|c|c|c|c|}
\hline$T_{\mathrm{min}}$ & $T_{\max }$ & $\begin{array}{r}T_{0} \\
\mathrm{~K}\end{array}$ & $\begin{array}{r}\Delta T \\
\mathrm{~K}\end{array}$ & $\begin{array}{r}\max \delta T \\
\mathrm{~K}\end{array}$ & $\begin{array}{l}\min \frac{\delta T}{\delta z} \\
\mathrm{~K} / \mathrm{km}\end{array}$ & $\begin{array}{r}\max \frac{\delta T}{\delta z} \\
\mathrm{~K} / \mathrm{km}\end{array}$ & $\begin{array}{r}\mathrm{H}_{2} \mathrm{O} \\
\mathrm{kg} / \mathrm{m}^{2}\end{array}$ & $\begin{array}{r}\mathrm{O}_{3} \\
\mathrm{DU}\end{array}$ \\
\hline 196.35 & 299.71 & 299.71 & 103.36 & 24.27 & -6.902 & 3.953 & 40.52 & 281.90 \\
\hline 215.70 & 294.21 & 294.21 & 78.51 & 27.75 & -6.671 & 2.469 & & 334.25 \\
\hline 215.20 & 272.07 & 272.07 & 56.87 & 15.64 & -6.130 & 3.032 & 8.33 & 375.98 \\
\hline 225.20 & 287.35 & 287.35 & 62.15 & 34.13 & -7.103 & 2.929 & 20.99 & 346.52 \\
\hline 211.87 & 258.07 & 257.24 & 46.20 & 12.94 & -6.966 & 2.445 & 4.08 & 373.21 \\
\hline 216.70 & 288.20 & 288.20 & 71.50 & 21.17 & -6.687 & 2.776 & 14.08 & 343.20 \\
\hline 200.00 & 273.59 & 247.28 & 73.59 & 20.69 & -7.500 & 6.593 & 2.26 & 209.77 \\
\hline 220.79 & 259.24 & 242.85 & 38.45 & 17.23 & -5.231 & 3.213 & 0.62 & 486.94 \\
\hline 204.00 & 272.60 & 258.10 & 68.60 & 21.16 & -7.129 & 5.071 & 5.85 & 338.26 \\
\hline 211.53 & 260.74 & 258.09 & 49.21 & 19.33 & -7.648 & 4.679 & 3.01 & 324.48 \\
\hline 213.89 & 275.78 & 275.78 & 61.89 & 9.90 & -9.257 & 2.184 & 6.99 & 359.45 \\
\hline 200.47 & 277.65 & 277.65 & 77.18 & 20.34 & -8.529 & 5.746 & 9.74 & 346.29 \\
\hline 199.00 & 280.36 & 280.02 & 81.36 & 23.78 & -8.075 & 6.375 & 9.94 & 275.58 \\
\hline 219.52 & 284.26 & 284.26 & 64.74 & 31.76 & -7.419 & 3.485 & 15.21 & 366.86 \\
\hline 207.26 & 284.72 & 284.72 & 77.46 & 12.71 & -6.895 & 2.433 & 25.95 & 266.82 \\
\hline 195.56 & 285.86 & 285.86 & 90.30 & 23.28 & -7.779 & 5.048 & 16 & 247.45 \\
\hline 199.45 & 302.54 & 302.54 & 103.09 & 12.58 & -8.8 & 3.797 & 50.75 & 240.26 \\
\hline 201.46 & 315.91 & 315.91 & 114.45 & 24.56 & -9.907 & 2.823 & 32.91 & 276.81 \\
\hline 213.82 & 258.41 & 252.19 & 44.59 & 13.01 & -6.565 & 2.475 & 2.35 & 495.21 \\
\hline 199.76 & 290.94 & 290.94 & 91.18 & 23.50 & -8.2 & 3.4 & 10.19 & 240 \\
\hline 214.19 & 285.11 & 285.11 & 70.92 & 16.86 & -8.886 & 3.328 & 12.94 & 334.80 \\
\hline 203.24 & 314.81 & 314.81 & 111.57 & 24.78 & -9.963 & 2.698 & 19.55 & 274.02 \\
\hline 198.56 & 299.50 & 299.50 & 100.94 & 23.73 & -7.807 & 3.372 & 22.25 & 236.81 \\
\hline 206.46 & 284.26 & 281.69 & 77.80 & 14.39 & -8.976 & 4.417 & 33.58 & 233.12 \\
\hline 218.30 & 292.39 & 292.39 & 74.09 & 32.74 & -7.595 & 3.307 & 37.05 & 272.91 \\
\hline 199.70 & 296.88 & 296.88 & 97.18 & 11.41 & -8 & 3.922 & & 260.43 \\
\hline 200.63 & 301.44 & 301.44 & 100.81 & 24.37 & -8.537 & 3.259 & 51.91 & 276.39 \\
\hline 197.91 & 301.84 & 301.84 & 103.93 & 12.39 & -8.568 & 3.711 & 59.43 & 259.79 \\
\hline 197.33 & 298.42 & 298.42 & 101.09 & 9.42 & -9.988 & 4.181 & 61.01 & 221.76 \\
\hline 199.91 & 301.63 & 301.63 & 101.72 & 23.99 & -7.420 & 3.540 & 67.49 & 245.08 \\
\hline 206.00 & 282.57 & 250 . & & & -6 & & 1.71 & 226 \\
\hline 202.55 & 299.35 & 299.35 & 96.80 & 24.40 & -7.929 & 3.489 & 26.60 & 261.29 \\
\hline 205.40 & 296.28 & 296.28 & 90.88 & 24.31 & -7.408 & 2.659 & 37.18 & 283.00 \\
\hline 210.42 & 283.59 & 283.59 & 73.17 & 13.89 & -7.314 & 3.268 & 12.00 & 288.84 \\
\hline 223.44 & 281.96 & 273.30 & 58.52 & 36.90 & -7.079 & 3.414 & 7.72 & 320.02 \\
\hline 214.58 & 279.10 & 254.20 & 64.52 & 20.45 & -6.5 & 2.6 & 3.73 & 342.09 \\
\hline 216.61 & 261.76 & 261.65 & & 21.13 & -7.0 & 3.0 & 5.03 & 373.46 \\
\hline 211.36 & 270.65 & 270.65 & 59.29 & 19.74 & -7.857 & 3.623 & 3.86 & 387.73 \\
\hline 214.96 & 254.31 & 254.15 & 39.35 & 11.22 & -8.033 & 3.053 & 2.29 & 421.43 \\
\hline 220.35 & 259.83 & 249.21 & 39.48 & 17.21 & -6.069 & 3.366 & 0.80 & 452.46 \\
\hline 215.94 & 255.70 & 253.28 & 39.76 & 17.37 & -6.786 & 4.519 & 1.93 & 475.69 \\
\hline 220.48 & 259.66 & 255.37 & 39.18 & 17.31 & -5.667 & 2.885 & 0.66 & 497.15 \\
\hline
\end{tabular}



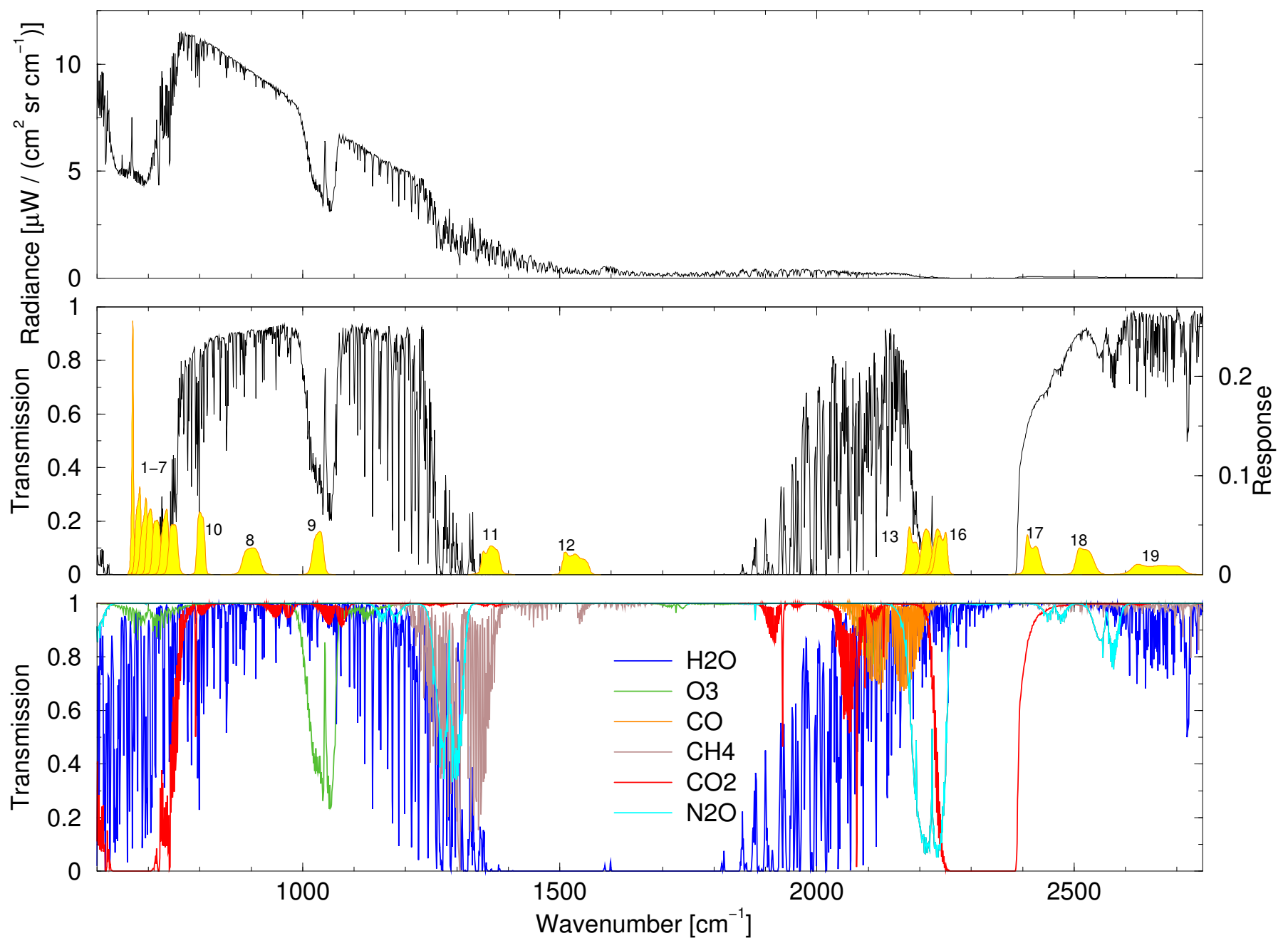

Figure 1: Atmospheric top-of-atmosphere spectral radiance (top) and the 19 HIRS channels (mid). The bottom plot indicates the contribution of the various absorbers to the total transmission also shown in the center plot. (Spectra calculated using the MODTRAN4 (MODerate resolution atmospheric TRANsmission) band model [57].)

have been parallelized in GARLIC [20, Section 3.3] using OpenMP (see http://openmp.org). With the optimized ARTS using predefined representative frequencies the computation of the 19 channel radiances for a single 43 level atmosphere takes about 10 minutes (CPU time for a single processor), whereas a truely monochromatic evaluation with a frequency grid similar to KOPRA requires more than 500 hours. On a multicore Intel-based machine, GARLIC needs about half a minute for this run. KOPRA executes this job in about 50 seconds on a single processor.

Fig. 3 shows the equivalent brightness temperatures for the six AFGL atmospheres and for the extreme atmospheres with the lowest and highest mean temperature, water and ozone content. The large variety of atmospheres is clearly translated into significantly different brightness temperature spectra. Note that the driest atmosphere as well as both extreme ozone atmospheres are very cold; in particular the ozone richest atmosphere \# 42 has a maximum temperature less than $260 \mathrm{~K}$. Furthermore, the data confirm that the first seven channels (as well as channels 13
- 16) indicate the atmospheric temperatures as prescribed by a weighting function analysis.

The comparison of equivalent brightness temperatures in Fig. 3 suggests a "perfect" agreement of the three codes. Indeed, the initial analysis 21] of temperature differences showed that for most (11 of 19) channels deviations are in the sub-Kelvin range, however, larger deviations of up to some Kelvin were found for some channels between GARLIC and ARTS or KOPRA. Later on, it was recognized that the contributions of $\mathrm{N}_{2}$ and $\mathrm{O}_{2}$ (lines and continua) have not been considered in the original GARLIC job setup.

The spectra shown in Fig. 3 have been generated running the codes in their default setting, i.e. with both lbl and continuum absorption contributions. However, one the main lessons learned from previous intercomparison studies, esp. [7, 11, has been the importance of consistent input data. However, apart from differences in the numerics, different continua implementations were recognized early on as a potential source of discrepancies. 


\section{Garand Atmospheres}
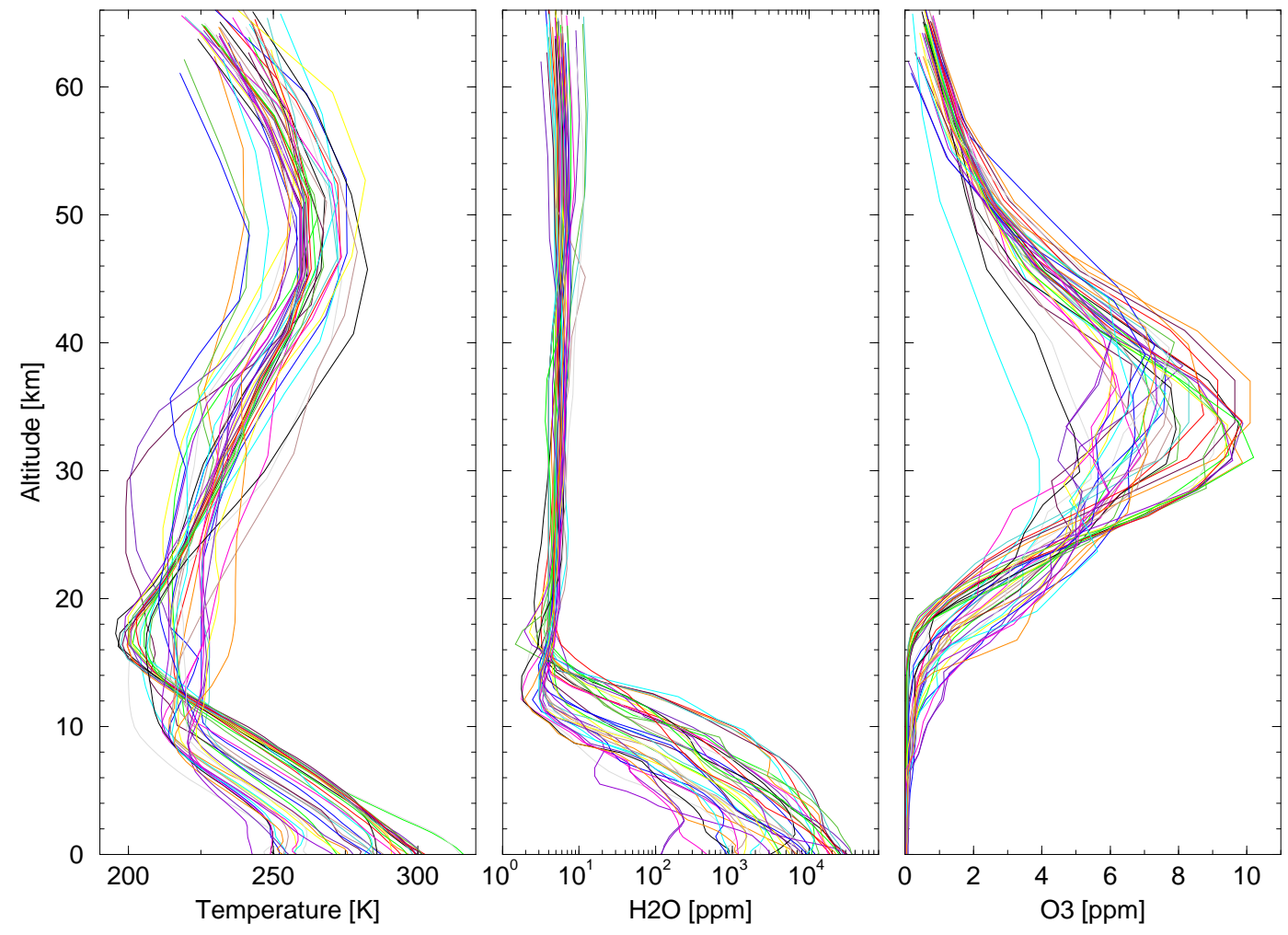

Figure 2: Temperature, $\mathrm{H}_{2} \mathrm{O}$, and $\mathrm{O}_{3}$ profiles of the 42 Garand atmospheres.

\subsection{First round: 43 level atmosphere with continua}

Fig. 4 depicts the pairwise differences of the equivalent brightness temperatures obtained by ARTS, GARLIC, and KOPRA as a function of the atmosphere number. First, note that except for a few channels the differences are less than $1 \mathrm{~K}$. There are only three channels (\# 3, 9 to 10) where all codes agree within half of a Kelvin for all atmospheres. ARTS and GARLIC also agree well in channels $1,2,4$, and 9, GARLIC is close to KOPRA in channels 3,9 , and 10, and KOPRA and ARTS match in channels $8,11,13-15$, and $18-19$. However, GARLIC has some substantial differences to ARTS and KOPRA for channel \# 11 centering at $1366 \mathrm{~cm}^{-1}$. On the other hand, KOPRA has the largest deviations to ARTS and GARLIC in channel \# 1, and large differences to ARTS in channel 6.

There appear to exist no common characteristics between the channels which would serve as explanation of the observed discrepancies. Channel \#1 at $669 \mathrm{~cm}^{-1}$ is in the center of the $\mathrm{CO}_{2} \nu_{2}$ band with significant absorption also by $\mathrm{H}_{2} \mathrm{O}$ and the corresponding weighting function peaking in the mid stratosphere (about $20 \mathrm{mb}$ ). Channels 13 and 17 are at the left and right flank of the $\mathrm{CO}_{2} \nu_{3}$ band with little absorption due to $\mathrm{H}_{2} \mathrm{O}$ and weighting function peaks in the lower troposphere.

Potential causes discussed comprise:
- Different line strength conversion schemes: In contrast to ARTS and KOPRA using the TIPS (Total Internal Partition Sum) 61] scheme, GARLIC evaluates the partition function according to the recipe utilized in the ATMOS data processing software 62. This issue has already been discussed in the context of the IRTMW3 intercomparison [11, indicating that small discrepancies (up to 10\%) between line strengths converted by the TIPS and ATMOS methods exist for a few molecules. Verdes et al. 63 have studied the impact of partition function data for the $\mathrm{mm} / \mathrm{sub}-\mathrm{mm}$ range; for the molecules considered there, the difference between the HITRAN-TIPS partition sums and the JPL partition sums 64 at 150 and $225 \mathrm{~K}$ was well less than one percent.

- Various lbl optimization schemes (wing truncation, weak line rejection, ... ): In view of the large discrepancies in channel 11, the GARLIC radiance has been recalculated using a "brute force" lbl algorithm instead of the "default" optimized multigrid code [48. KOPRA uses an optimized irregular altitude dependent frequency grid for calculation of absorption cross sections and integration of the radiative transfer equation [see also 60]. However, for all atmospheres the changes were well below one Kelvin and did not have 

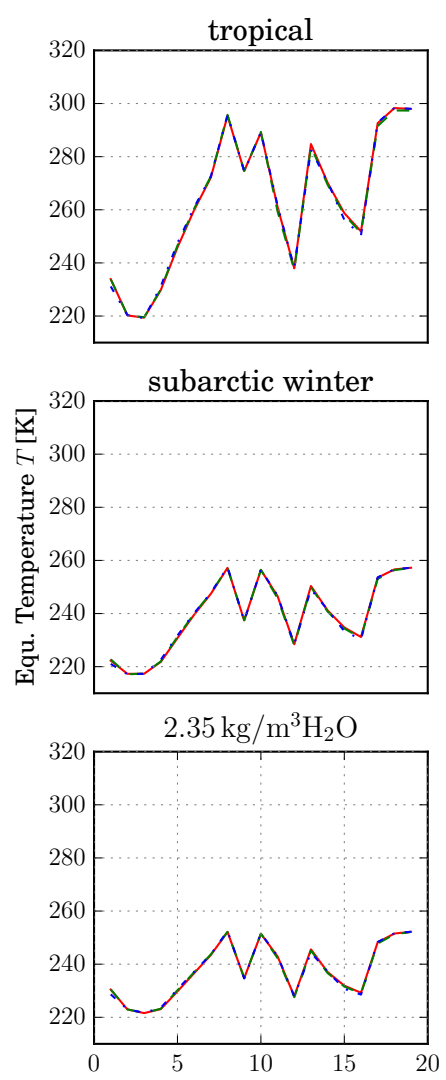

midlatitude summer

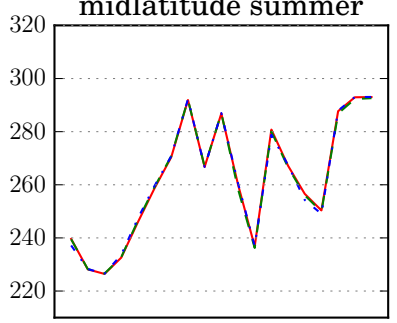

US standard

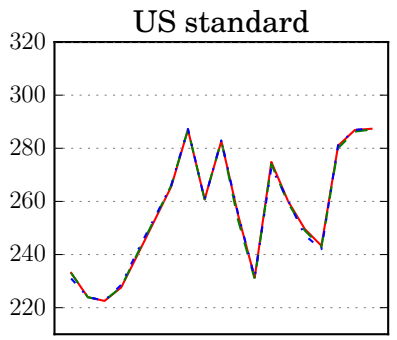

$67.5 \mathrm{~kg} / \mathrm{m}^{3} \mathrm{H}_{2} \mathrm{O}$

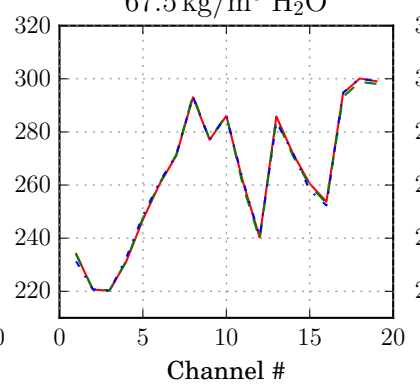

midlatitude winter

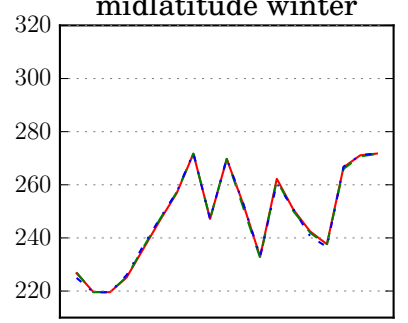

$T_{\text {mean }}=227 \mathrm{~K}$

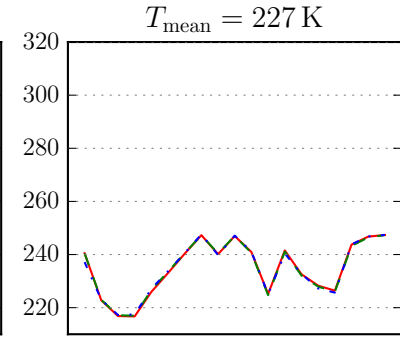

$226 \mathrm{DU} \mathrm{O}_{3}$

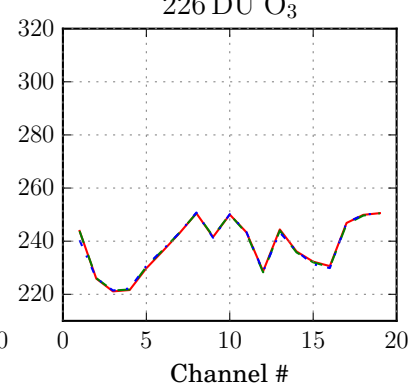

subarctic summer
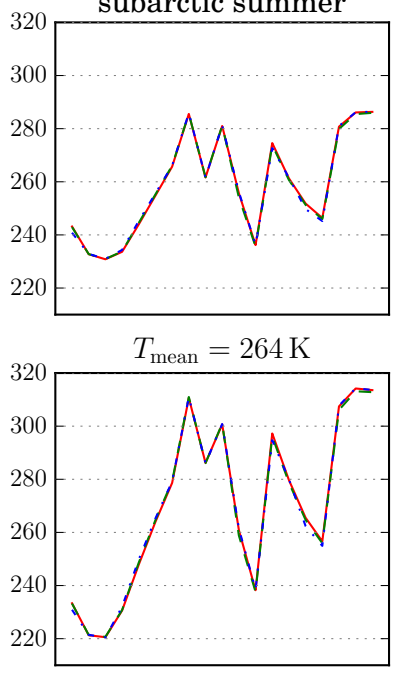

$487 \mathrm{DU} \mathrm{O}_{3}$

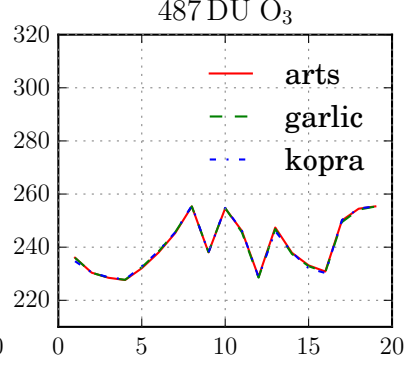

Figure 3: Brightness temperatures as a function of channel number for the Garand atmospheres $1-6,7$ and 18 (min and max temperature), 19 and 30 (min and max water), 31 and 42 (min and max ozone). Continuum included, 43 atmospheric levels.

any impact on the brightness temperature differences.

- Evaluation of path integrals: As noted in Section 2 . the three codes use different approaches to evaluate the optical depth (2) and Schwarzschild (1) integrals: ARTS assumes a piecewise linear variation of the absorption and approximates the Planck function in a layer by the mean of the level values. GARLIC uses numerical quadratures, where the atmospheric state is represented by discrete values representing selected levels. Contrary to that, KOPRA uses representative averages of atmospheric state values for each layer, similar as recommended by Curtis [51] and Godson 52. These representative averages are calculated as mass-weighted averages along the actual ray path segment through a layer. The related numerical integration involves sublayering.

- Continua $\left(\mathrm{H}_{2} \mathrm{O}, \mathrm{CO}_{2}, \ldots\right)$ : the different versions of the continua implemented in the three codes were identified as the most likely reason for the discrepancies. This assumption is further supported by Fig. 5 showing that the brightness temperature differences are roughly increasing with the atmosphere's water content. In particular, the GARLIC and KOPRA (old CKD continuum) vs. ARTS (newer MT-CKD continuum) deviations are large and increasing in channel \# 11 at the left end of the $\mathrm{H}_{2} \mathrm{O} 6.3 \mu \mathrm{m}$ band where the two continua differ substantially (see also, e.g. 65, Fig. 1]). Thus, a recalculation of the radiances without continua contributions was initiated.

For an assessment of the discrepancies a comparison of high resolution monochromatic spectra appeared to be an option. However, for technical/practical reasons it is difficult to run the three radiation codes with numerically identical frequency grids. Even small differences in the exact grid points cause large local radiance deviations, due to the highly structured spectra. A high resolution monochromatic view of the results is therefore misleading. Additionally, the nature of the different models make it difficult (if not impossible) to calculate on a common grid as it would no longer be the original model as used here: ARTS uses an optimized coarse grid with a weighted mean of representative frequencies 33, whereas GARLIC uses a uniform dense grid [20, Section 3.2], and the automatically determined non-equidistant grid is a typical feature of KOPRA. 


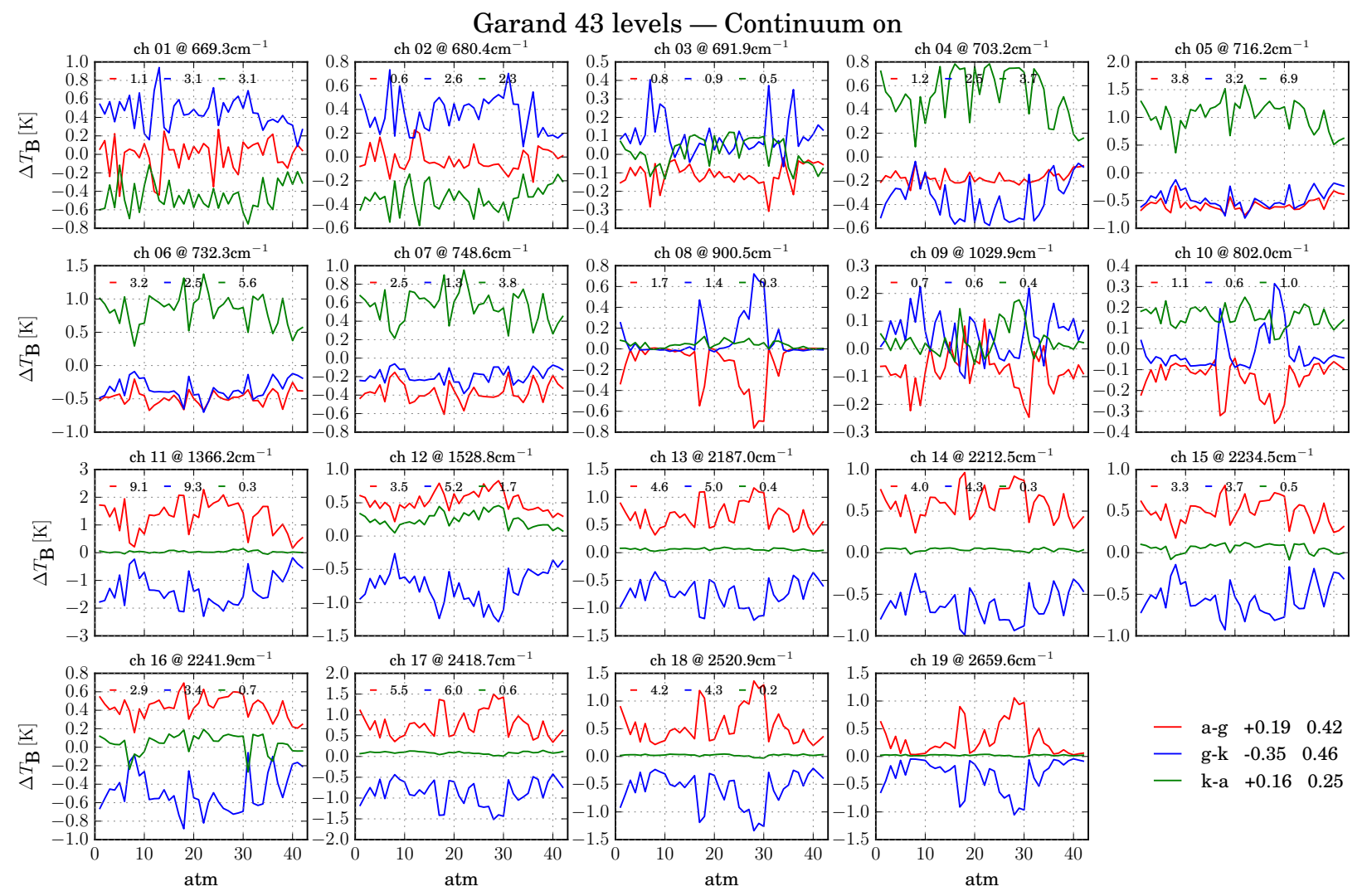

Figure 4: Difference brightness temperatures as a function of atmospheric profile number, channel by channel: 43 level atmosphere, continuum included. The numbers in the legend indicate the norm of the mutual difference vector. The numbers in the lower right corner are the mean difference and mean absolute difference.
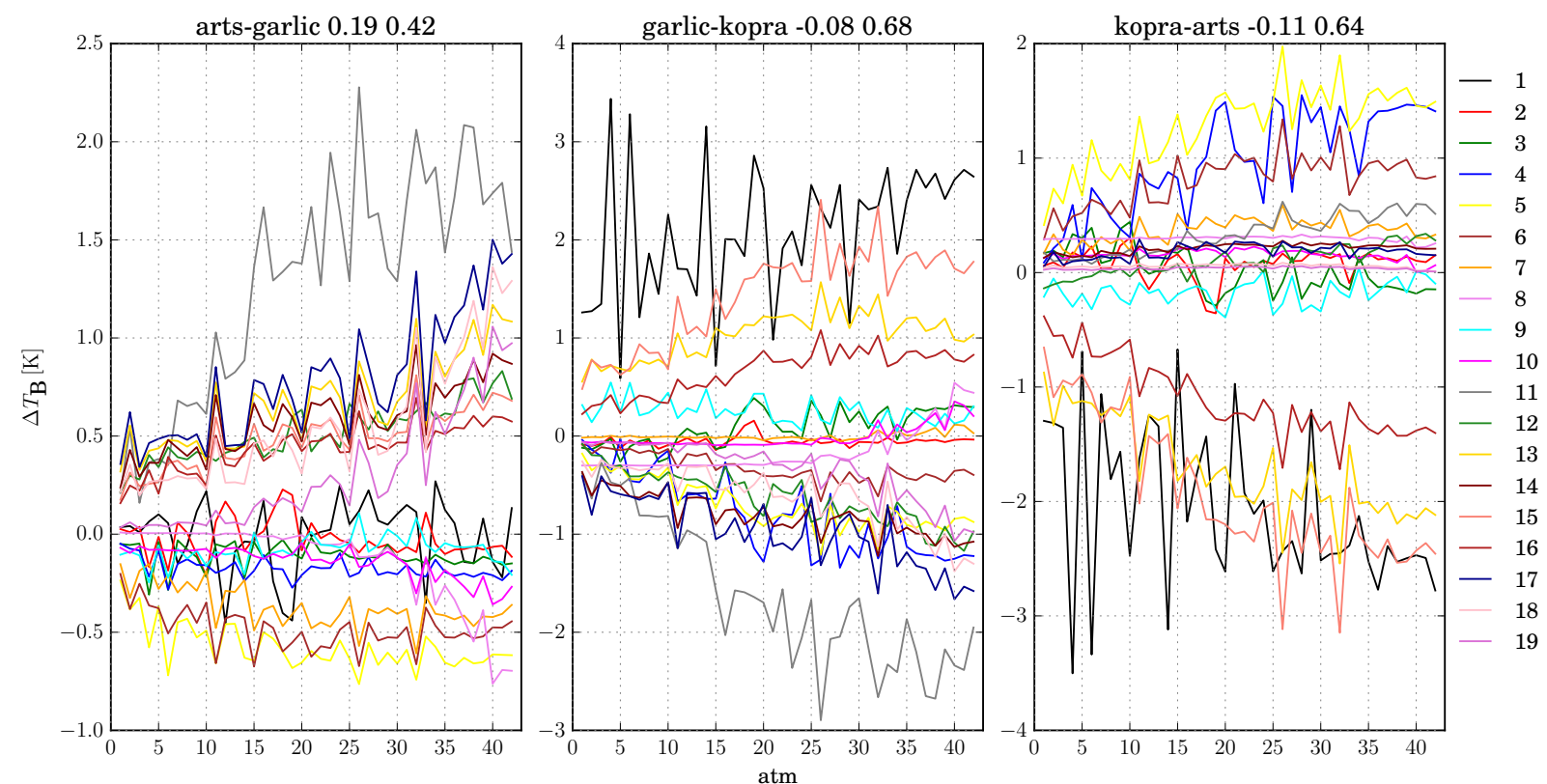

Figure 5: Brightness temperature differences as a function of atmospheric profile number sorted by vertical column density of water for all channels: 43 level atmosphere, continuum included. The legends indicate the channel number. The numbers in the subplot titles give the mean difference and mean absolute difference. 


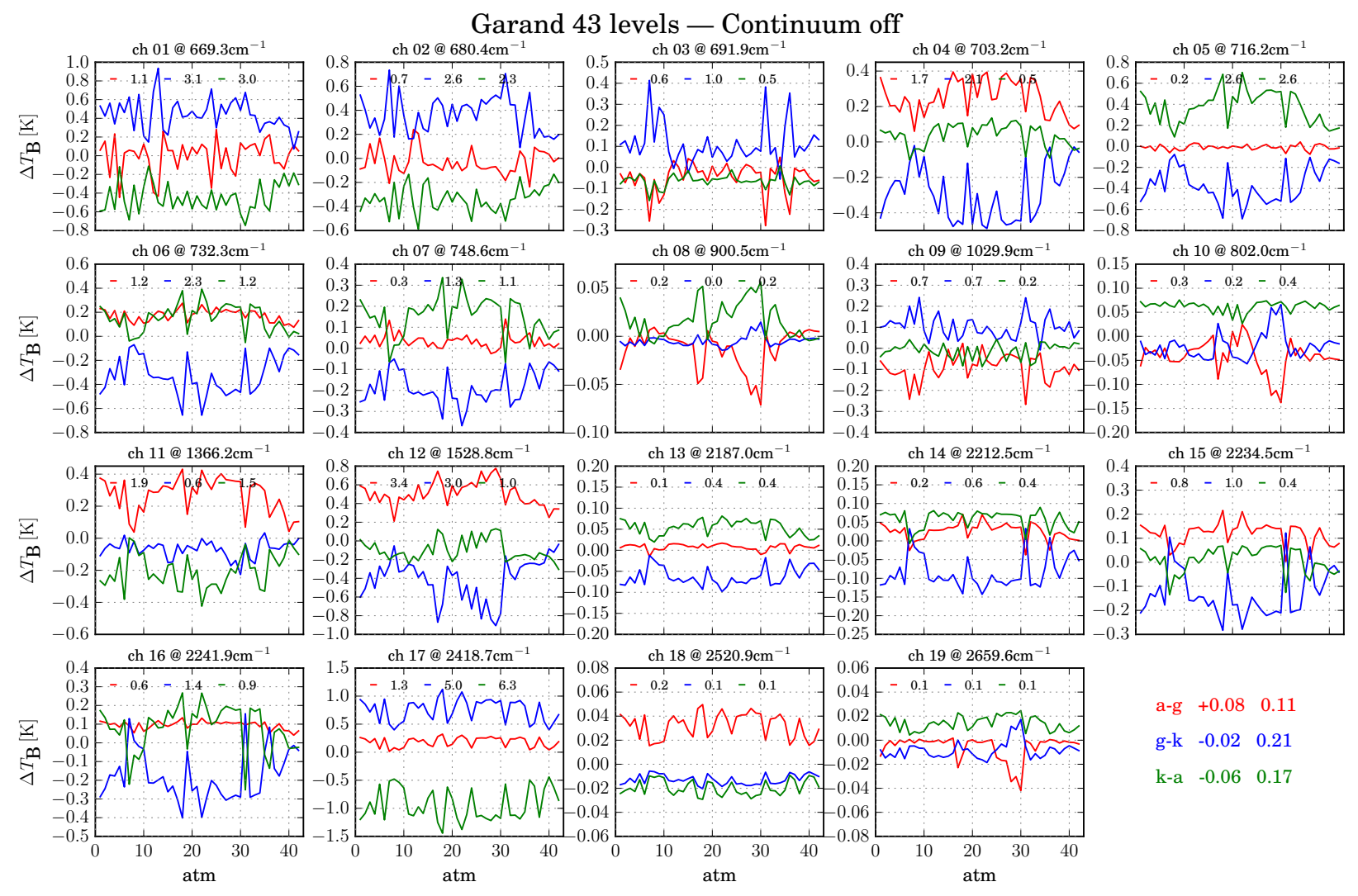

Figure 6: Brightness temperature differences as a function of atmospheric profile number for all channels: 43 level atmosphere, no continuum.

\subsection{Second round: 43 level atmosphere without continua}

A comparison of Fig. 6 with Fig. 4 clearly indicates the large impact of the continua. Except for one channel (\# 17) the mutual differences are now well below $1 \mathrm{~K}$ for all atmospheres. At $2419 \mathrm{~cm}^{-1}$ (the short wavelength edge of the $\mathrm{CO}_{2} \nu_{3}$ band) KOPRA shows somewhat larger differences around one Kelvin to ARTS and GARLIC that are attributed to the different treatment of $\mathrm{CO}_{2}$ line wings. For the channels 1, 2, 5, 6, and 12 differences for some of the atmospheres exceed $0.5 \mathrm{~K}$, whereas in the window channels \# 8 (around $900 \mathrm{~cm}^{-1}$ ) and 18 and 19 (2521 and $2660 \mathrm{~cm}^{-1}$ ) differences are smaller than $0.1 \mathrm{~K}$. The two channels on the long wavelength edge of the $\mathrm{CO}_{2}$ band also show good agreement of the three codes. In all channels (except \# 17) where ARTS and KOPRA already agreed well with continuum, the differences were further reduced. As shown at http://rtweb.aer.com/continuum_frame. html, the $\mathrm{H}_{2} \mathrm{O}$ foreign and self continua have a maximum around $1500-1700 \mathrm{~cm}^{-1}$, however, the change from the CKD to the MT-CKD continuum is more prominent in the left and right flanks. Accordingly, the large discrepancy of GARLIC to ARTS and KOPRA in channel \# 11 around $1366 \mathrm{~cm}^{-1}$ (Fig. 4) is significantly reduced without continua, whereas the $1529 \mathrm{~cm}^{-1}$ region is not much affected. Likewise, the reduction of the ARTS vs. KOPRA differences (e.g. channels 4 to 6 ) could indicate different (MT-)CKD versions.
Fig. 7 reveals that the changes (at least the large changes) due to the continuum are similar for all models. In particular, for all atmospheres the equivalent brightness temperature is changed by several Kelvin in channel \# 17 at the shortwave edge of the $\mathrm{CO}_{2}$ band around $2419 \mathrm{~cm}^{-1}$ when the continuum is "switched on". Furthermore the three codes are sensitive in channels 8 and 10 for the atmospheres 17 and $28-30$ characterized by water columns larger than $50 \mathrm{~kg} / \mathrm{m}^{2}$ (cf. Tab. 1).

Recalling the discussion of possible causes for the discrepancies in the previous subsection suggests that the different path integration schemes might be responsible for some of the observed differences. As a first test we compared the two schemes for the Schwarzschild integral implemented in GARLIC: The change from the "linear in optical depth" to the "exponential in optical depth" quadrature scheme results in brightness temperature differences up to $0.16 \mathrm{~K}$. The largest difference is for channel 1 and the Garand atmosphere \# 13 characterized by the second largest $\delta T / \delta z$; channel 12 has large differences for atmospheres \# 17, 26, and 29. Note that for all channels and all atmospheres the radiance computed by the "linear $\tau$ " approximation is larger than for "exponential $\tau$ ". For the 85 level atmospheres discussed below the two integration schemes result in differences up to $0.08 \mathrm{~K}$. These differences can be interpreted as an inherent uncertainty of about $0.1 \mathrm{~K}$ due to path integration. 

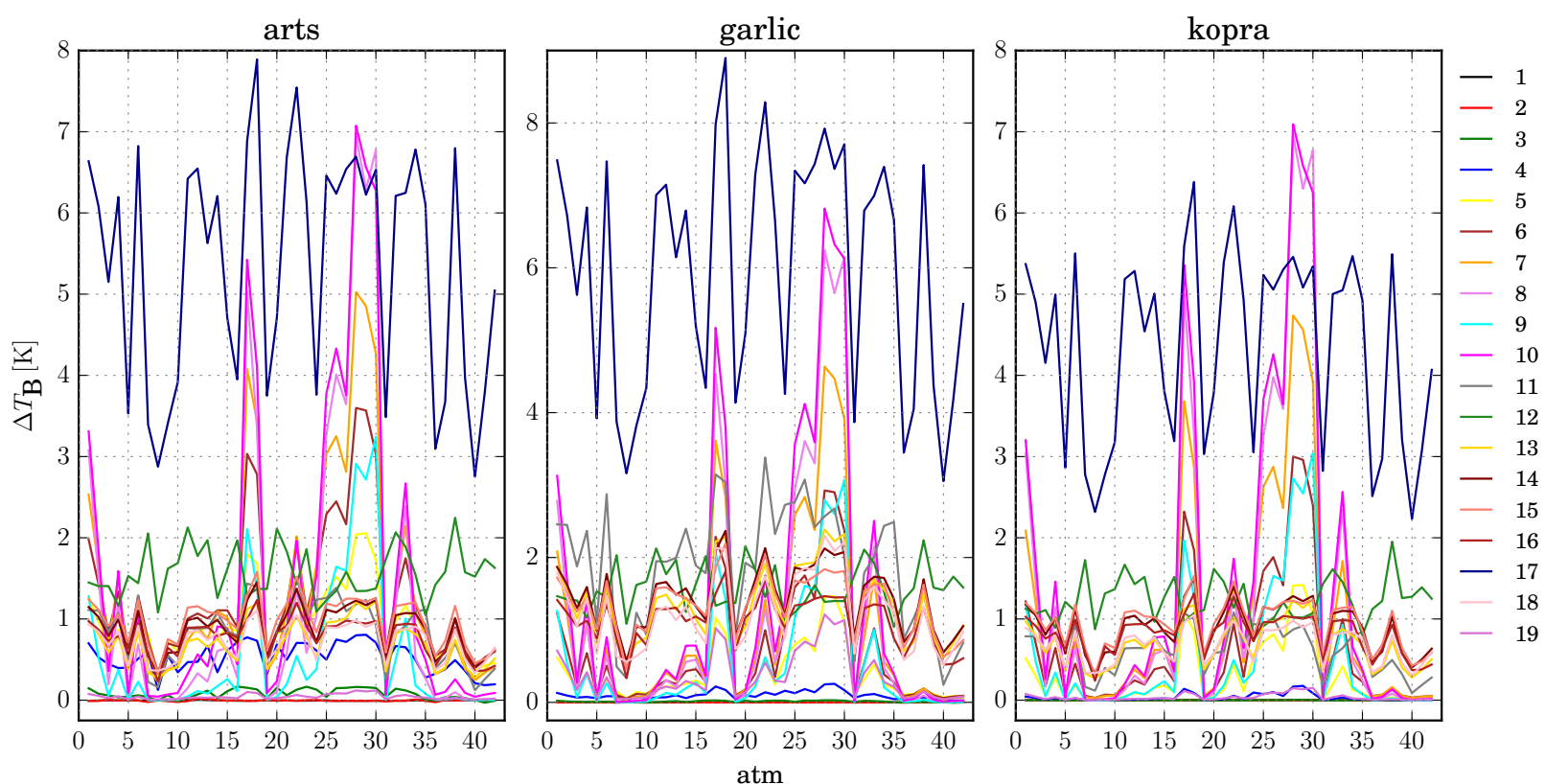

Figure 7: Brightness temperature differences $T_{\mathrm{B}}^{0}-T_{\mathrm{B}}^{1}$ due to continuum (43 level atmosphere).

ARTS uses the altitude levels of the input profiles for layering as long as the level distance along the line-of-sight is smaller than a given (layer) path length. For level distances longer than that given path lengths, the calculation will be done on the finer path length. Tests with finer level spacing of $250 \mathrm{~m}$ and $50 \mathrm{~m}$, respectively, showed that the results slightly changed for the narrow altitude grids but differences between the models remained within the range of the observed results. This issue was also reduced by the use of the higher resolved input profiles with 85 and 169 levels, respectively.

Another way to assess the treatment of the inhomogeneous atmosphere is to plot the differences vs. some measure of the temperature variability. Fig. 8 indicates that increasing temperature differences tend to larger brightness temperature differences. In order to judge this conjecture better, a dense "copy" of the Garand atmospheric profile with 85 levels has been generated, where the additional 42 levels were obtained by piecewise cubic Hermite interpolation 66 with new altitude grid points halfway between the original ones.

\subsection{Third round: 85 and 169 level atmosphere without continua}

Fig. 9 indicates that for a given model the change from 43 to 85 levels can result in differences up to half a Kelvin, with KOPRA showing the smallest sensitivity to the number of levels. The mutual brightness temperature differences in channels 1, 2, 3, and 12 shown in Fig. 10 are clearly smaller than those in Fig. 6, whereas the differences in channels $4,6-8$, and 15 are slightly smaller, and changes can also be seen in channels $9-11$. In channels 11 and 13 the differences to KOPRA are somewhat larger, and in channel 9 the KOPRA-ARTS residuum increases. Except for channel \# 17 the differences are smaller than half a Kelvin, and in the three window channels $(8,18$, and 19) they are less than $0.1 \mathrm{~K}$.

A further bisection of the layers does not further reduce the differences significantly. Apparently other issues beyond the numerics of path integration become important, in particular, the different approximations used to speed up the time critical lbl computation.

\subsection{Final round: 85 level atmosphere with continua}

Having demonstrated the importance of consistent continua data and the impact of the altitude discretization on smaller residuals, the 85 levels Garand atmospheres are finally used to evaluate spectra with the continuum "switched on" again. Fig. 11 demonstrates that the changes from 43 to 85 levels for "continuum on" are largely consistent with those for "continuum off". Channels 5, 6,18 , and 19 are only minimally affected by the change of the atmospheric layering, whereas some channels show reduced differences with the fine grid, most prominently channel 1 (as in the "continuum off" case). The largest difference of slightly more than $2 \mathrm{~K}$ can be observed between ARTS and GARLIC in channel 11, consistent with the results discussed in subsection 4.1

\section{Discussion and Conclusions}

An intercomparison of thermal infrared spectra modeled by the three independent lbl codes ARTS, GARLIC, and KOPRA has been presented assuming a nadir viewing HIRS-like instrument with 19 channels from 670 to $2700 \mathrm{~cm}^{-1}$. To capture the wide variety of atmospheric 

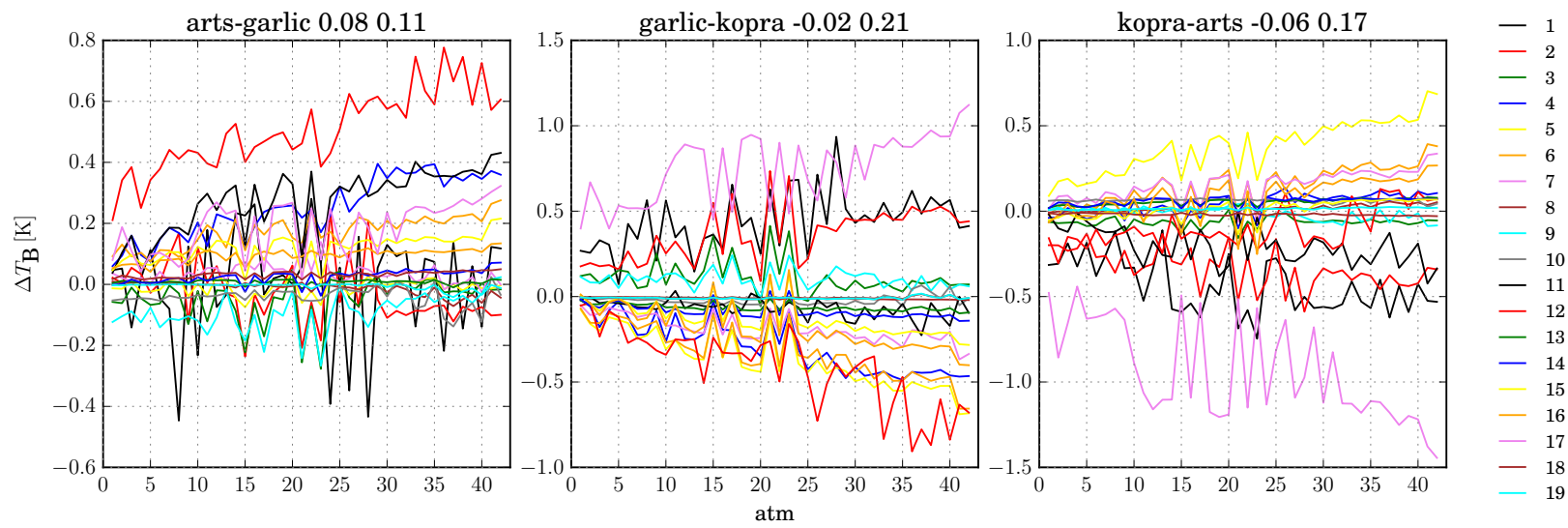

Figure 8: Difference brightness temperatures as a function of atmospheric profile number sorted by maximum $\Delta T=T_{\max }-T_{\min }: 43$ level atmosphere, continuum off.
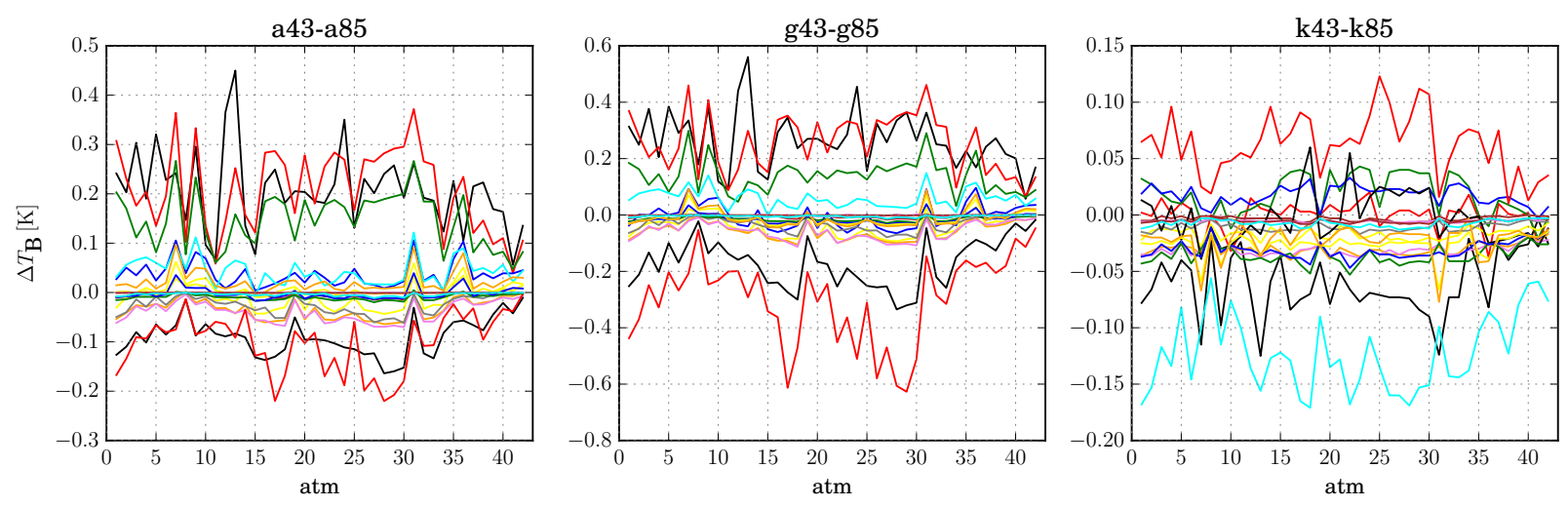

Figure 9: Brightness temperature differences as a function of atmospheric profile number for all channels: 43 level vs. 85 level atmospheres. (No continuum)

conditions, the 42 Garand atmospheres have been considered. Except for a few channels and/or atmospheres, the codes generally agree quite well with deviations less than one Kelvin. Averaging over all atmospheres (Fig. 12), discrepancies are smaller than half a Kelvin except for a few channels, mostly due to the choice of the continuum model used.

Figure 2 of Garand et al. [6] indicates the largest spread of brightness temperature differences in channel 9 at $1028 \mathrm{~cm}^{-1}$, and the smallest spread for channel 10 at $796 \mathrm{~cm}^{-1}$. In contrast, for the six channels similar to Garand's our Fig. 6 reveals the largest spread for channel 5 and the smallest spread for channel 10.

In contrast to previous intercomparisons none of the participating codes has been selected as the reference. Obviously a mutual comparison becomes infeasible for a large number of codes, i.e. even for four codes there would be six combinations. On the other hand, with only two codes under investigation [as in 19], there is no way to "decide who is correct". The objective of this comparison has not been to identify any "superior" code. The analysis has clearly indicated that there are not two perfectly agreeing codes for all channels and atmospheres, and none of the codes appears as an outlier relative to the other two. In some cases ARTS and GARLIC agreed nicely with KOPRA being offside, in other cases GARLIC and KOPRA were consistent or ARTS and KOPRA. Note that the agreement of two or even three codes does not necessarily imply their correctness. With the continuum switched on, some of the differences were as large as a few Kelvin, whereas without continuum the maximum difference were mostly in the sub-Kelvin range. In some of the window channels the three codes had an agreement better than $0.1 \mathrm{~K}$.

One of the lessons learned in previous intercomparisons has been the importance of consistent/common input data. Accordingly, the discrepancies between ARTS, GARLIC, and KOPRA due to the different continua did not come as a surprise and one can argue the value of this particular comparison. However, running the codes with their default setting has been the very first exercise of this project. This was intentionally done so, invoking that for the user it is important to know how the codes perform in their nominal setup. Nevertheless, the comparison clearly showed that despite different continua the codes agree remarkably well except for a few channels, indicating that these data variants do not necessarily produce different radiance spectra. 


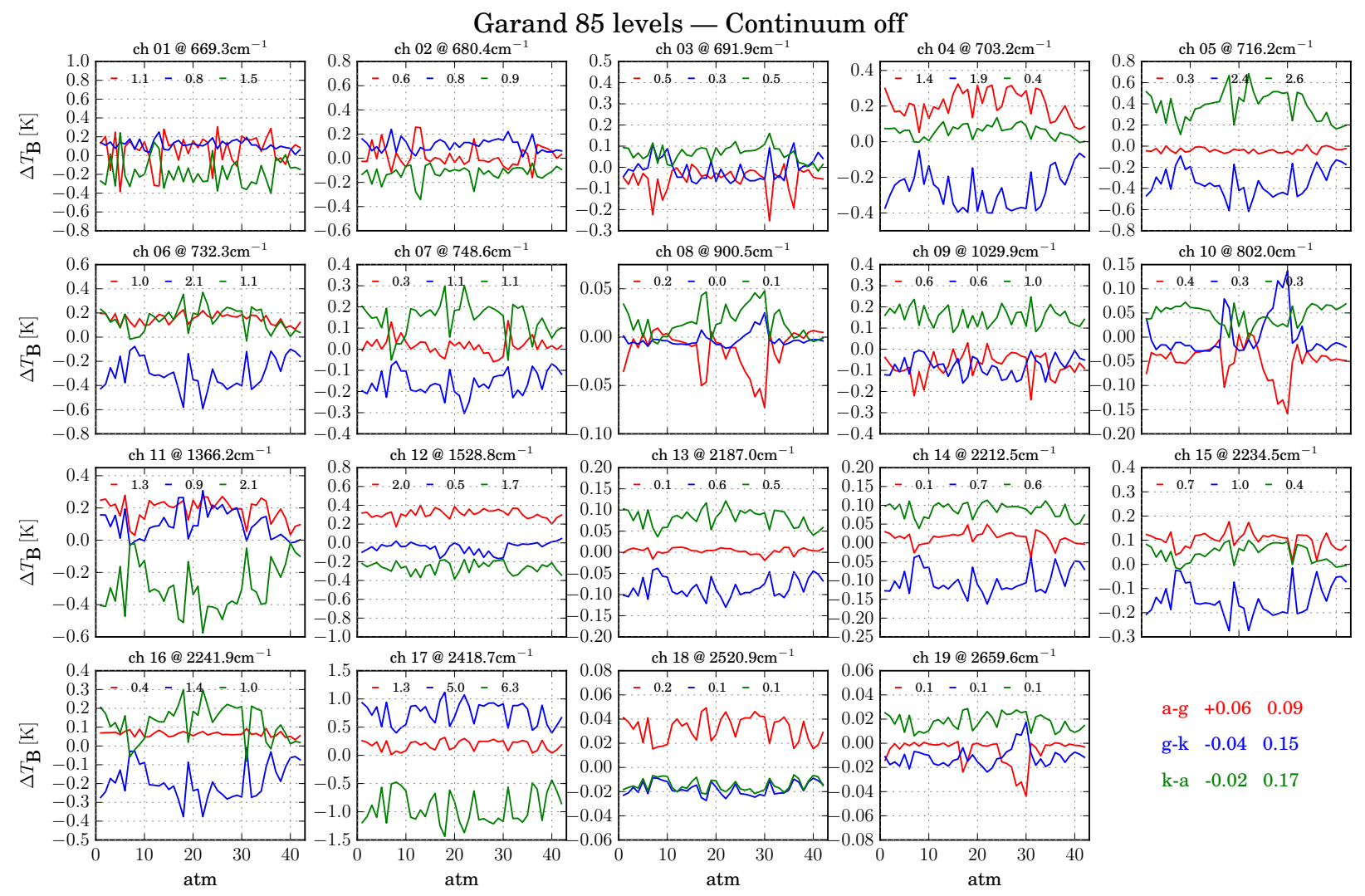

Figure 10: Brightness temperature differences as a function of atmospheric profile number: 85 level atmosphere, no continuum.
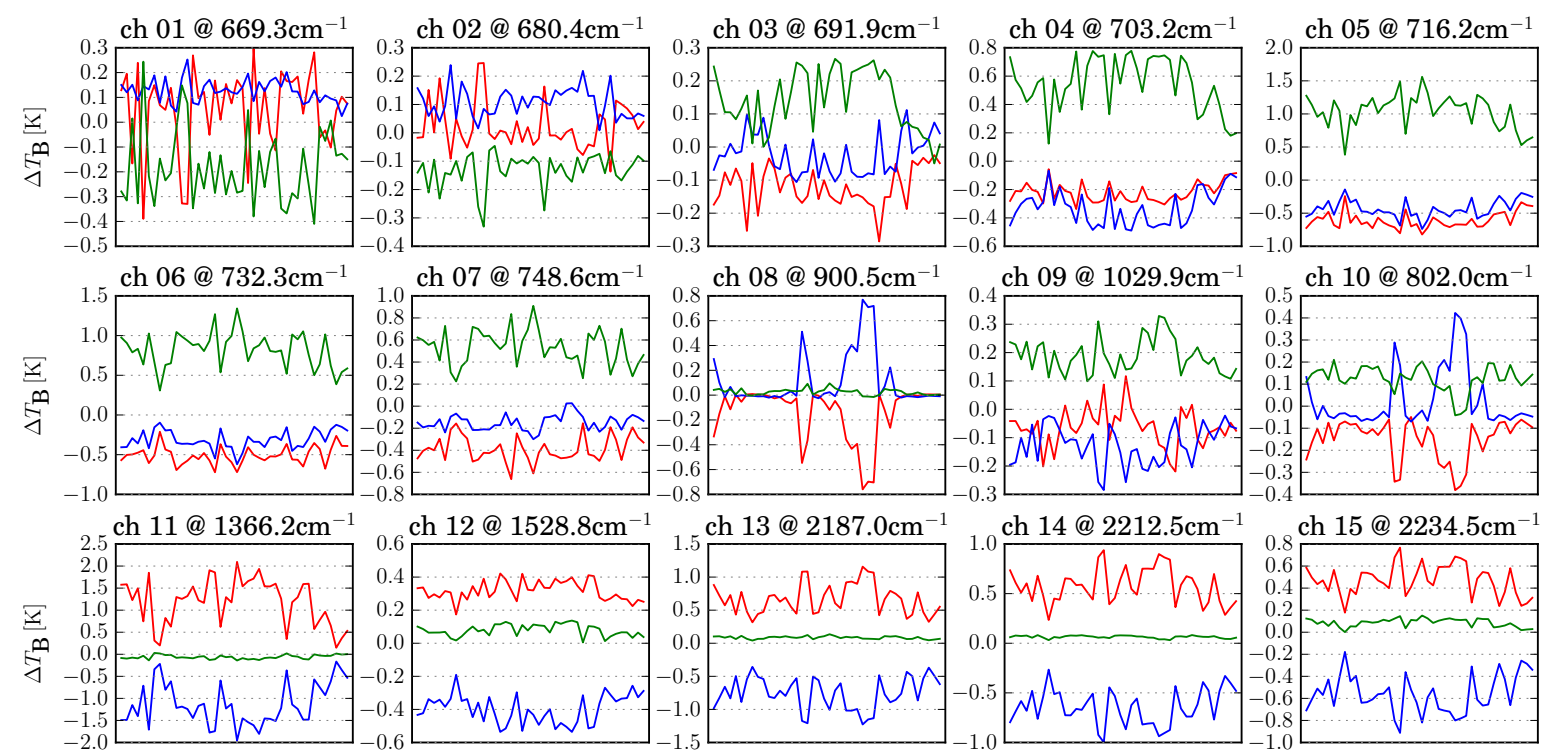

ch $13 @ 2187.0 \mathrm{~cm}^{-1}$

ch $14 @ 2212.5 \mathrm{~cm}^{-1}$

$\operatorname{ch} 15 @ 2234.5 \mathrm{~cm}^{-1}$
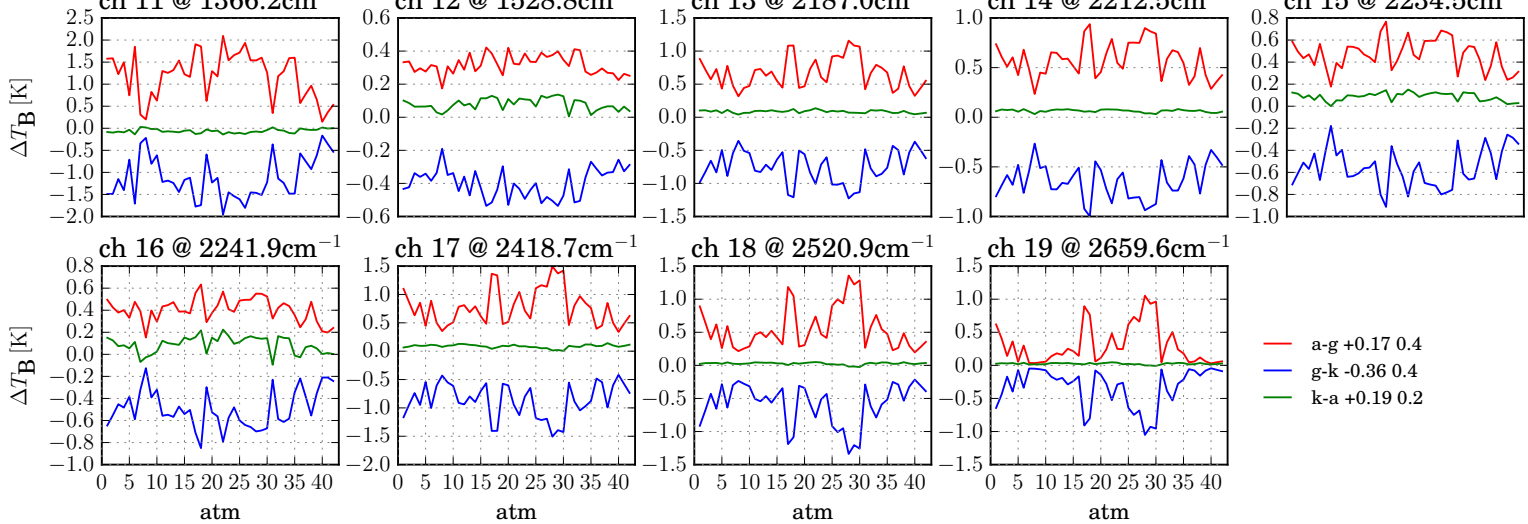

ch $18 @ 2520.9 \mathrm{~cm}^{-1}$

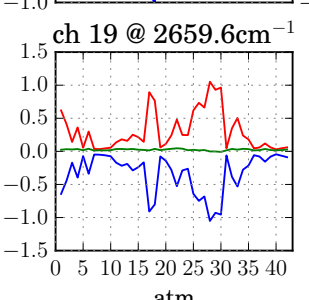

- a-g +0.170 .4$

- $\mathrm{g}-\mathrm{k}-0.360 .4$

Figure 11: Brightness temperature differences as a function of atmospheric profile number: 85 level atmosphere, with continuum. 


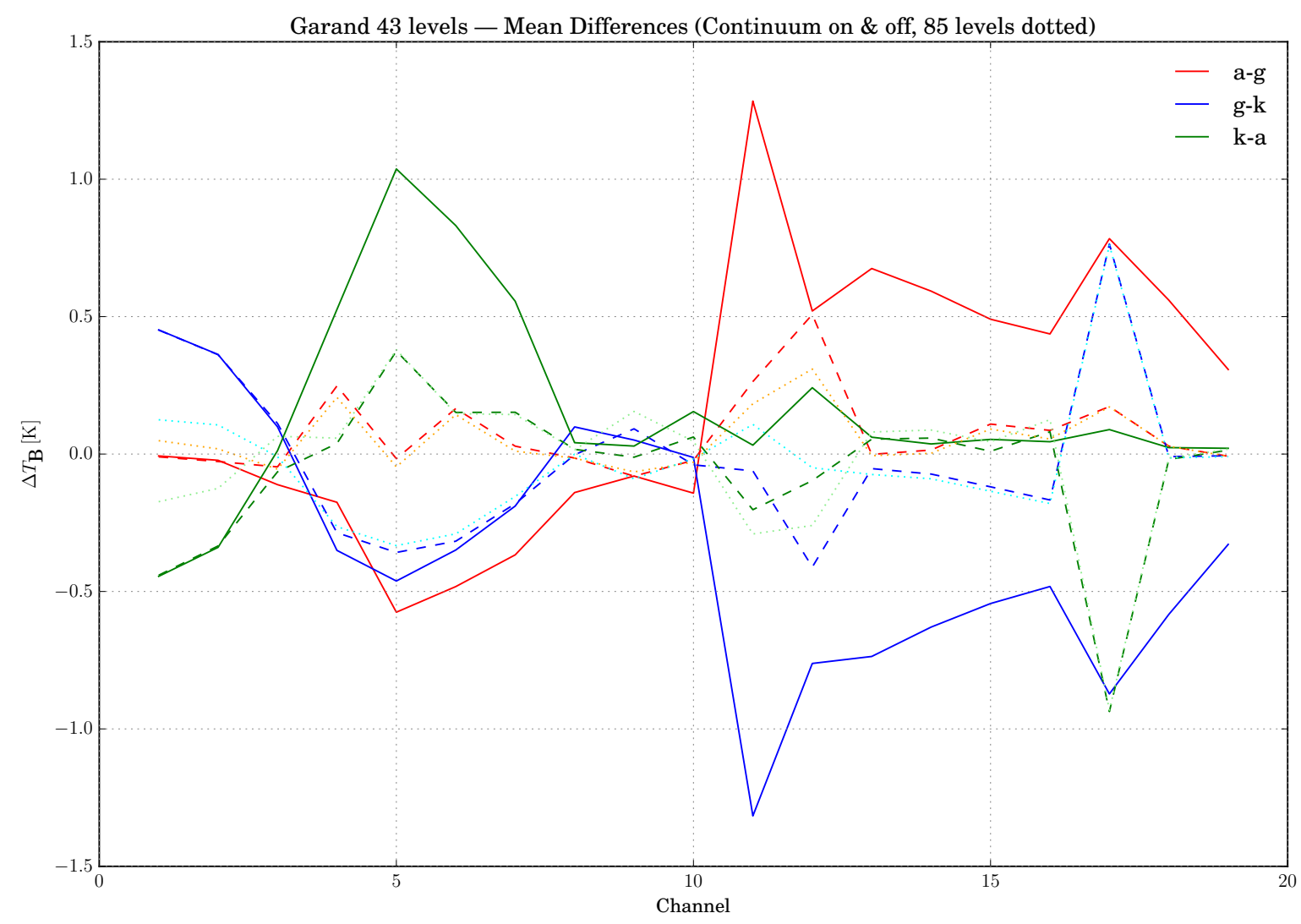

Figure 12: Difference brightness temperatures averaged over all atmospheres as a function of channel number: Solid lines: 43 level atmosphere with continuum; dashed lines: without continuum; dotted lines: 85 level atmosphere without continuum.

Esposito et al. 67] noted that "typical differences introduced by the two $\mathrm{H}_{2} \mathrm{O}$ continuum models are of one order of magnitude less than typical differences arising from different line parameters."

Efficient yet accurate lbl modeling of molecular absorption is a big challenge for high resolution models in view of the million to billions of line transitions [68, 69] and the terabyte to petabyte of remote sensing data to be processed. Hence, lbl models in general and the three codes considered here differ substantially in their approach of lbl optimization (see subsections 2.2 - 2.4). However, if a certain line profile such as Voigt is adopted (i.e. ignoring effects to collisional narrowing, line mixing, or speeddependence of the relaxation rate [cf., e.g. 29]), any optimization can be easily checked against a brute-force reference calculation. Thus, implementation of lbl absorption can be considered as a kind of well-defined recipe with only a few physics-related free parameters such as line wing cutoff wavenumber. Furthermore, the correct modeling of lbl cross sections can be validated by comparison with laboratory spectra recorded in an absorption cell with well defined pressure, temperature, and composition. Likewise, laboratory studies could help to characterize the continuum appropriately [e.g. 35, 65, 70].

The results of the calculations presented in subsections 4.2 and 4.3 suggest that the details of the lbl implementa- tion have a small impact on the consistency of the models. We interpret these differences up to a few tenths of a Kelvin as a result of the various lbl optimizations. However, an in depth assessment of these differences would have required editing of the codes and has been considered as beyond the scope of this study. Note that in the IRTMW3 intercomparison [11 the radiative transfer calculations have been performed in two stages: In "exercise 3" all participating models used absorption coefficients provided by ARTS, and the resulting differences were much smaller compared to "exercise 4" where both absorption and radiative transfer were calculated individually.

In contrast to the lbl absorption modeling, the discretization of the atmospheric profiles and the evaluation of the integrals (1) and (2) are more subtle, and the "correct" methodology cannot easily be determined by model intercomparisons. Obviously, the path integration schemes used by the three codes are all approximations; when sublayers are introduced as in ARTS and KOPRA, assumptions on the "correct" / appropriate interpolation of the integrand and/or absorption cross sections or coefficients have to be made. Sophisticated closure experiments [e.g. 71, 72 would be required. Nevertheless, doubling or even quadrupling of the atmospheric layers (see subsection 4.3) can be used for an assessment of this issue, i.e. despite the 
different approaches used by the three codes the differences are generally reduced to some tenths of a Kelvin.

In conclusion, the analysis has clearly indicated that none of the codes is "perfect". However, a perfect (lbl) atmospheric radiative transfer code is probably impossible for a variety of reasons: The Voigt line profile (4) adopted here for convenience is clearly an approximation, and despite the recent consensus with respect to a more sophisticated line shape (the Hartmann-Tran profile [cf. 29]) it is likely that further subtleties will show up with even more sensitive instruments. Even with a perfectly well known line shape the numerical treatment is far from clear, e.g. the cutoff in the line wings [e.g. 73]. Moreover, a proper description of the continuum [70] and collision-induced absorption 74 is far from satisfactory. (Multiple) scattering [23 25] and non-LTE [8, 50, 75] further complicate matters. For the modeling of spectral observations a precise knowledge of the instrumental line shape function and calibration can also be a limiting factor. Uncertainties due to the instrument's field-of-view (a.k.a. point spread function) and the horizontal variability of the atmosphere are especially important for limb sounding observations. Finally, because of the ever increasing amount of data (line data, observations, ...) approximations are mandatory.

This study has shown that with respect to the numerical implementation of radiative transfer schemes a level of maturity has been gained that subtle differences of the underlying physical models become relevant. In view of the large range of equivalent brightness temperature observed in Earth's atmosphere (roughly $215 \leq T_{\mathrm{B}} \leq 300 \mathrm{~K}$ ), compare Fig. 3), an agreement within one Kelvin (or a tenth of a Kelvin for some channels) is equivalent to about one percent discrepancies and is considered as satisfactory.

Acknowledgements. Sebastian Gimeno García and Ajil Kottayil helped in the early phase of the project. We would like to thank Patrick Eriksson and Jana Mendrok (Chalmers, Gothenburg), Gabi Stiller and Michael Höpfner (KIT Karlsruhe) for numerous discussions. F.S. would like to thank Jana Mendrok and Stefan Buehler for funding a short stay in Kiruna and gratefully acknowledges financial support by the Deutsche Forschungsgemeinschaft — DFG (projects SCHR 1125/3-1).

\section{References}

[1] Ronald F. Boisvert, Ronald Cools, and Bo Einarsson. Assessment of accuracy and reliability. In Bo Einarsson, editor, Accuracy and Reliability in Scientific Computing, chapter 2. SIAM, Philadelphia, PA, 2005.

[2] H. Fischer, G.P. Anderson, Thomas von Clarmann, S.A. Clough, M.T. Coffey, A. Goldman, and F.X. Kneizys. Intercomparison of transmittance and radiance algorithms (ITRA), Report of the Limb-Group of the ITRA Workshop at the University of Maryland, March 1986. KfK-Report 4349, Kernforschungszentrum Karlsruhe, Germany, 1988.

[3] R.G. Ellingson and Y. Fouquart. The intercomparison of radiation codes in climate models: An overview. J. Geophys. Res., 96:8925-8927, 1991. doi: 10.1029/90JD01618.
[4] N. Glatthor, M. Hoepfner, G.P. Stiller, T. von Clarmann, A. Dudhia, G. Echle, B. Funke, and F. Hase. Intercomparison of the KOPRA and the RFM radiative transfer codes. In Satellite Remote Sensing of Clouds and the Atmosphere IV, volume 3867. SPIE, 1999. doi: 10.1117/12.373077.

[5] B. Soden, S. Tjemkes, J. Schmetz, R. Saunders, J. Bates, B. Ellingson, R. Engelen, L. Garand, D. Jackson, G. Jedlovec, T. Kleespies, D. Randel, P. Rayer, E. Salathe, D. Schwarzkopf, N. Scott, B. Sohn, S. de Souza-Machado, L. Strow, D. Tobin, D. Turner, P. van Delst, and T. Wehr. An intercomparison of radiation codes for retrieving upper-tropospheric humidity in the $63-\mathrm{mm}$ band: A report from the first GVaP workshop. Bull. Am. Met. Soc., 81(4):797-808, 2000. doi: 1520-0477(2000)081<0797:AIORCF $\rangle 2.3 . \mathrm{CO} ; 2$.

[6] L. Garand, D.S. Turner, M. Larocque, J. Bates, S. Boukabara, P. Brunel, F. Chevallier, G. Deblonde, R. Engelen, M. Hollingshead, D. Jackson, G. Jedlovec, J. Joiner, T. Kleespies, D.S. McKague, L.M. McMillen, J.-.L. Moncet, J.R. Pardo, P.J. Rayer, E. Salathé, R. Saunders, N.A. Scott, P. Van Delst, and H. Woolf. Radiance and Jacobian intercomparison of radiative transfer models applied to HIRS and AMSU channels. J. Geophys. Res., 106(D20):24017-24031, 2001. doi: 10.1029/2000JD000184.

[7] T. von Clarmann, M. Höpfner, B. Funke, M. López-Puertas, A. Dudhia, V. Jay, F. Schreier, M. Ridolfi, S. Ceccherini, B.J. Kerridge, J. Reburn, and R. Siddans. Modeling of atmospheric mid-infrared radiative transfer: The AMIL2DA algorithm intercomparison experiment. J. Quant. Spectrosc. \& Radiat. Transfer, 78:381-407, 2002. doi: 10.1016/S0022-4073(02) 00262-5.

[8] Thomas von Clarmann, A. Dudhia, D. P. Edwards, B. Funke, M. Höpfner, B. Kerridge, V. Kostsov, A. Linden, M. LópezPuertas, and Yu. Timofeyev. Intercomparison of radiative transfer codes under non-local thermodynamic equilibrium conditions. J. Quant. Spectrosc. \& Radiat. Transfer, 107(D22):4631, 2002. doi: 10.1029/2001JD001551.

[9] S.A. Tjemkes, T. Patterson, R. Rizzi, M.W. Shephard, S.A. Clough, M. Matricardi, J.D. Haigh, M. Höpfner, S. Payan, A. Trotsenko, N. Scott, P. Rayer, J.P. Taylor, C. Clerbaux, L.L. Strow, S. DeSouza-Machado, D. Tobin, and R. Knuteson. The ISSWG line-by-line inter-comparison experiment. J. Quant. Spectrosc. \& Radiat. Transfer, 77(4):433-453, 2003. doi: 10.1016/S0022-4073(02)00174-7.

[10] D.P. Kratz, M.G. Mlynczak, C.J. Mertens, H. Brindley, L.L. Gordley, J. Martin-Torres, F.M. Miskolczi, and D.D. Turner. An inter-comparison of far-infrared line-by-line radiative transfer models. J. Quant. Spectrosc. ES Radiat. Transfer, 90:323-341, 2005. doi: 10.1016/j.jqsrt.2004.04.006.

[11] C. Melsheimer, C. Verdes, S.A. Buehler, C. Emde, P. Eriksson, D.G. Feist, S. Ichizawa, V.O. John, Y. Kasai, G. Kopp, N. Koulev, T. Kuhn, O. Lemke, S. Ochiai, F. Schreier, T.R. Sreerekha, M. Suzuki, C. Takahashi, S. Tsujimaru, and J. Urban. Intercomparison of general purpose clear sky atmospheric radiative transfer models for the millimeter/submillimeter spectral range. Radio Sci., 40:RS1007, 2005. doi: 10.1029/ 2004RS003110.

[12] S.A. Buehler, N. Courcoux, and V.O. John. Radiative transfer calculations for a passive microwave satellite sensor: Comparing a fast model and a line-by-line model. J. Geophys. Res., 111: D20304, 2006. doi: 10.1029/2005JD006552.

[13] R. Saunders, P. Rayer, P. Brunel, A. von Engeln, N. Bormann, L. Strow, S. Hannon, S. Heilliette, Xu Liu, F. Miskolczi, Y. Han, G. Masiello, J.-L. Moncet, Gennady Uymin, V. Sherlock, and D. S. Turner. A comparison of radiative transfer models for simulating Atmospheric Infrared Sounder (AIRS) radiances. $J$. Geophys. Res., 112:D01S90, 2007. doi: 10.1029/2006JD007088.

[14] L.A. Sromovsky, P.M. Fry, V. Boudon, A. Campargue, and A. Nikitin. Comparison of line-by-line and band models of nearIR methane absorption applied to outer planet atmospheres. 
Icarus, 218(1):1-23, 2012. doi: 10.1016/j.icarus.2011.12.008.

[15] J. Yang, J. Leconte, E.T. Wolf, C. Goldblatt, N. Feldl, T. Merlis, Y. Wang, D.D.B. Koll, F. Ding, F. Forget, and D.S. Abbot. Differences in water vapor radiative transfer among $1 \mathrm{D}$ models can significantly affect the inner edge of the habitable zone. ApJ, 826(2):222, 2016. doi: 10.3847/0004-637X/826/2/222.

[16] G.P. Stiller, T. von Clarmann, B. Funke, N. Glatthor, F. Hase, M. Höpfner, and A. Linden. Sensitivity of trace gas abundances retrievals from infrared limb emission spectra to simplifying approximations in radiative transfer modelling. $J$. Quant. Spectrosc. \& Radiat. Transfer, 72:249-280, 2002. doi: 10.1016/S0022-4073(01)00123-6.

[17] S.A. Buehler, P. Eriksson, T. Kuhn, A. von Engeln, and C. Verdes. ARTS, the atmospheric radiative transfer simulator. J. Quant. Spectrosc. \& Radiat. Transfer, 91:65-93, 2005. doi: $10.1016 /$ j.jqsrt.2004.05.051.

[18] P. Eriksson, S.A. Buehler, C. Davis, C. Emde, and O. Lemke. ARTS, the atmospheric radiative transfer simulator, version 2 . J. Quant. Spectrosc. \& Radiat. Transfer, 112(10):1551-1558, 2011. doi: 10.1016/j.jqsrt.2011.03.001.

[19] M. Milz. ARTS IR application. In Open ARTS Community Workshop 2010, June 2010.

[20] F. Schreier, S. Gimeno García, P. Hedelt, M. Hess, J. Mendrok, M. Vasquez, and J. Xu. GARLIC - a general purpose atmospheric radiative transfer line-by-line infrared-microwave code: Implementation and evaluation. J. Quant. Spectrosc. \& Radiat. Transfer, 137:29-50, 2014. doi: 10.1016/j.jqsrt.2013.11.018.

[21] F. Schreier, S. Gimeno García, M. Milz, A. Kottayil, M. Höpfner, T. von Clarmann, and G. Stiller. Intercomparison of three microwave/infrared high resolution line-by-line radiative transfer codes. In Robert F. Cahalan and Jürgen Fischer, editors, Radiation Processes in the Atmosphere and Ocean (IRS2012): Proceedings of the International Radiation Symposium (IRC/IAMAS), volume 1531 of AIP Conference Proceedings, pages 119-122, 2013. doi: 10.1063/1.4804722.

[22] M.I. Mishchenko. Directional radiometry and radiative transfer: The convoluted path from centuries-old phenomenology to physical optics. J. Quant. Spectrosc. \& Radiat. Transfer, 146: 4-33, 2014. doi: 10.1016/j.jqsrt.2014.02.033.

[23] R.M. Goody and Y.L. Yung. Atmospheric Radiation - Theoretical Basis. Oxford University Press, second edition, 1989.

[24] Kuo-Nan Liou. An Introduction to Atmospheric Radiation. Academic Press, second edition, 2002.

[25] W. Zdunkowski, T. Trautmann, and A. Bott. Radiation in the Atmosphere - A Course in Theoretical Meteorology. Cambridge University Press, 2007.

[26] K.P. Shine, I.V. Ptashnik, and G. Rädel. The water vapour continuum: Brief history and recent developments. Surv. Geophys., 33(3-4):535-555, 2012. doi: 10.1007/s10712-011-9170-y.

[27] B.H. Armstrong. Spectrum line profiles: The Voigt function. J. Quant. Spectrosc. \& Radiat. Transfer, 7:61-88, 1967. doi: 10.1016/0022-4073(67)90057-X.

[28] P.L. Varghese and R.K. Hanson. Collisional narrowing effects on spectral line shapes measured at high resolution. Appl. Opt., 23(14):2376-2385, 1984. doi: 10.1364/AO.23.002376.

[29] J. Tennyson, P.F. Bernath, A. Campargue, A.G. Császár, L. Daumont, R.R. Gamache, J.T. Hodges, D. Lisak, O.V. Naumenko, L.S. Rothman, H. Tran, N.F. Zobov, J. Buldyreva, C.D. Boone, M.D. De Vizia, L. Gianfrani, J.-M. Hartmann, R. McPheat, D. Weidmann, J. Murray, N.H. Ngo, and O.L. Polyansky. Recommended isolated-line profile for representing high-resolution spectroscopic transitions (IUPAC technical report). Pure Appl. Chem., 86(12):1931-1943, 2014. doi: 10.1515/pac-2014-0208.

[30] I.E. Gordon, L.S. Rothman, et al. The HITRAN2016 molecular spectroscopic database. J. Quant. Spectrosc. \& Radiat. Transfer, 203:3 - 69, 2017. doi: 10.1016/j.jqsrt.2017.06.038.
[31] S.A. Buehler, J. Mendrok, P. Eriksson, A. Perrin, R. Larsson, and O. Lemke. ARTS, the atmospheric radiative transfer simulator - version 2.2, the planetary toolbox edition. Geosci. Model Dev. Disc., 2017:1-28, 2017. doi: 10.5194/gmd-2017-229.

[32] G. Holl, S.A. Buehler, J. Mendrok, and A. Kottayil. Optimised frequency grids for infrared radiative transfer simulations in cloudy conditions. J. Quant. Spectrosc. \& Radiat. Transfer, 113:2124-2134, 2012. doi: 10.1016/j.jqsrt.2012.05.022.

[33] S.A. Buehler, V.O. John, A. Kottayil, M. Milz, and P. Eriksson. Efficient radiative transfer simulations for a broadband infrared radiometer — combining a weighted mean of representative frequencies approach with frequency selection by simulated annealing. J. Quant. Spectrosc. \& Radiat. Transfer, 111 (4):602-615, 2010. doi: 10.1016/j.jqsrt.2009.10.018.

[34] S.A. Clough, F.X. Kneizys, and R. Davies. Line shape and the water vapor continuum. Atm. Res., 23:229-241, 1989. doi: 10.1016/0169-8095(89)90020-3.

[35] E.J. Mlawer, V.H. Payne, J.-L. Moncet, J.S. Delamere, M.J. Alvarado, and D.C. Tobin. Development and recent evaluation of the MT-CKD model of continuum absorption. Philosophical Transactions of the Royal Society A: Mathematical, Physical and Engineering Sciences, 370(1968):2520-2556, 2012. doi: 10. 1098/rsta.2011.0295.

[36] S. Buehler, P. Eriksson, and O. Lemke. Absorption lookup tables in the radiative transfer model ARTS. J. Quant. Spectrosc. \& Radiat. Transfer, 112(10):1559-1567, 2011. doi: 10.1016/j. jqsrt.2011.03.008.

[37] S.A. Buehler, A. von Engeln, E. Brocard, V.O. John, T. Kuhn, and P. Eriksson. Recent developments in the line-by-line modeling of outgoing longwave radiation. J. Quant. Spectrosc. 86 Radiat. Transfer, 98:4467-457, 2006. doi: 10.1016/j.jqsrt.2005. 11.001

[38] R. Pincus, A. Beljaars, S.A. Buehler, G. Kirchengast, F. Ladstaedter, and J.S. Whitaker. The representation of tropospheric water vapor over low-latitude oceans in (re-)analysis: Errors, impacts, and the ability to exploit current and prospective observations. Surveys in Geophysics, 38(6):1399-1423, 2017. doi: 10.1007/s10712-017-9437-z.

[39] F. Schreier, S. Gimeno García, M. Vasquez, and J. Xu. Algorithmic vs. finite difference Jacobians for infrared atmospheric radiative transfer. J. Quant. Spectrosc. \& Radiat. Transfer, 164:147-160, 2015. doi: 10.1016/j.jqsrt.2015.06.002.

[40] P. Hochstaffl, F. Schreier, G. Lichtenberg, and S. Gimeno García. Validation of carbon monoxide total column retrievals from SCIAMACHY observations with NDACC/TCCON ground-based measurements. Remote Sensing, 10(2):223, 2018. doi: $10.3390 / \mathrm{rs} 10020223$

[41] J. Xu, F. Schreier, G. Wetzel, A. de Lange, M. Birk, T. Trautmann, A. Doicu, and G. Wagner. Performance assessment of balloon-borne trace gas sounding with the Terahertz channel of TELIS. Remote Sensing, 10(2):315, 2018. doi: 10.3390/ rs10020315.

[42] F. Schreier, S. Städt, P. Hedelt, and M. Godolt. Transmission spectroscopy with the ACE-FTS infrared spectral atlas of Earth: A model validation and feasibility study. Molec. Astrophysics, 2018. doi: 10.1016/j.molap.2018.02.001. in press.

[43] F. Schreier and S. Gimeno García. Py4CAtS - Python tools for line-by-line modelling of atmospheric radiative transfer. In Robert F. Cahalan and Jürgen Fischer, editors, Radiation Processes in the Atmosphere and Ocean (IRS2012): Proceedings of the International Radiation Symposium (IRC/IAMAS), volume 1531 of AIP Conference Proceedings, pages 123-126. American Institute of Physics, 2013. doi: 10.1063/1.4804723.

[44] Aleksandra Borysow. Collision-induced absorption in the infrared: A data base for modelling planetry and stellar atmospheres, 2002. URL http://www.astro.ku.dk/ aborysow/ programs/index.html

[45] J. Humlíček. Optimized computation of the Voigt and complex 
probability function. J. Quant. Spectrosc. \& Radiat. Transfer, 27:437-444, 1982. doi: 10.1016/0022-4073(82)90078-4.

[46] J.A.C. Weideman. Computation of the complex error function. SIAM J. Num. Anal., 31:1497-1518, 1994. doi: 10.1137/ 0731077.

[47] F. Schreier. Optimized implementations of rational approximations for the Voigt and complex error function. J. Quant. Spectrosc. \& Radiat. Transfer, 112(6):1010-1025, 2011. doi: 10.1016/j.jqsrt.2010.12.010.

[48] F. Schreier. Optimized evaluation of a large sum of functions using a three-grid approach. Comp. Phys. Comm., 174:783-802, 2006. doi: 10.1016/j.cpc.2005.12.015.

[49] H. Fischer, M. Birk, C. Blom, B. Carli, M. Carlotti, T. von Clarmann, L. Delbouille, A. Dudhia, D. Ehhalt, M. Endemann, J. M. Flaud, R. Gessner, A. Kleinert, R. Koopman, J. Langen, M. López-Puertas, P. Mosner, H. Nett, H. Oelhaf, G. Perron, J. Remedios, M. Ridolfi, G. Stiller, and R. Zander. MIPAS: an instrument for atmospheric and climate research. Atm. Chem. Phys., 8(8):2151-2188, 2008. doi: 10.5194/acp-8-2151-2008.

[50] B. Funke, M. López-Puertas, M. García-Comas, M. Kaufmann, M. Höpfner, and G.P. Stiller. GRANADA: A Generic RAdiative traNsfer AnD non-LTE population algorithm. J. Quant. Spectrosc. \& Radiat. Transfer, 113(14):1771 - 1817, 2012. doi: 10.1016/j.jqsrt.2012.05.001.

[51] A.R. Curtis. A statistical model for water-vapour absorption. Quart. J. R. Met. Soc., 78:638-640, 1952.

[52] W.L. Godson. The evaluation of infra-red radiative fluxes due to atmospheric water vapour. Quart. J. R. Met. Soc., 79:367-379, 1953.

[53] M. Kuntz. A new implementation of the Humlicek algorithm for the calculation of the Voigt profile function. $J$ Quant. Spectrosc. \& Radiat. Transfer, 57:819-824, 1997. doi: 10.1016/S0022-4073(96)00162-8.

[54] W.J. Lafferty, A.M. Solodov, A. Weber, W.B. Olson, and J.M. Hartmann. Infrared collision-induced absorption by $\mathrm{N}_{2}$ near $4.3 \mu \mathrm{m}$ for atmospheric applications: measurements and empirical modeling. Appl. Opt., 35(30):5911-5917, 1996. doi: 10.1364/AO.35.005911.

[55] F. Thibault, V. Menoux, R. Le Doucen, L. Rosenmann, J.-M. Hartmann, and Ch. Boulet. Infrared collision-induced absorption by $\mathrm{O}_{2}$ near $6.4 \mu \mathrm{m}$ for atmospheric applications: measurements and empirical modeling. Appl. Opt., 36(3):563-567, 1997. doi: 10.1364/AO.36.000563.

[56] G. Echle and M. Höpfner. The Karlsruhe Optimized and Precise Radiative Transfer Algorithm (KOPRA). volume FZKA 6487 of Wissenschaftliche Berichte, chapter VIII. Parameterization of continua caused by gaseous constituents, pages 89 93. Forschungszentrum Karlsruhe, 2000. URL http://www . imk-asf.kit.edu/downloads/sat/kopra_docu_part08.pdf.

[57] A. Berk, G.P. Anderson, P.K. Acharya, L.S. Bernstein, J.H. Chetwynd, M.W. Matthew, E.P. Shettle, and S.M. AdlerGolden. MODTRAN4 user's manual. Technical report, Air Force Research Laboratory, Mass., USA, June 1999.

[58] G.P. Anderson, S.A. Clough, F.X. Kneizys, J.H. Chetwynd, and E.P. Shettle. AFGL atmospheric constituent profiles (0 - $120 \mathrm{~km}$ ). Technical Report TR-86-0110, AFGL, 1986.

[59] L.S. Rothman et al. The HITRAN 2004 molecular spectroscopic database. J. Quant. Spectrosc. E Radiat. Transfer, 96:139-204, 2005. doi: 10.1016/j.jqsrt.2004.10.008.

[60] M. Kuntz and M. Höpfner. Efficient line-by-line calculation of absorption coefficients. J. Quant. Spectrosc. $\&$ Radiat. Transfer, 63:97-114, 1999. doi: 10.1016/S0022-4073(98)00140-X.

[61] J. Fischer, R.R. Gamache, A. Goldman, L.S. Rothman, and A. Perrin. Total internal partition sums for molecular species in the 2000 edition of the HITRAN database. J. Quant. Spectrosc. \& Radiat. Transfer, 82:401-412, 2003. doi: 10.1016/ S0022-4073(03)00166-3.
[62] R.H. Norton and C.P. Rinsland. ATMOS data processing and science analysis methods. Appl. Opt., 30:389-400, 1991. doi: 10.1364/AO.30.000389.

[63] C.L. Verdes, S.A. Buehler, A. Perrin, J.-M. Flaud, J. Demaison, G. Wlodarczak, J.-M. Colmont, G. Cazzoli, and C. Puzzarini. A sensitivity study on spectroscopic parameter accuracies for a $\mathrm{mm} /$ sub-mm limb sounder instrument. J. Mol. Spectrosc., 229 (2):266-275, 2005. doi: 10.1016/j.jms.2004.09.014.

[64] H.M. Pickett, R.L. Poynter, E.A. Cohen, M.L. Delitsky, J.C. Pearson, and H.S.P. Müller. Submillimeter, millimeter, and microwave spectral line catalog. J. Quant. Spectrosc. \& Radiat. Transfer, 60:883-890, 1998. doi: 10.1016/S0022-4073(98) 00091-0.

[65] D. J. Paynter and V. Ramaswamy. An assessment of recent water vapor continuum measurements upon longwave and shortwave radiative transfer. J. Geophys. Res., 116:D20302, 2011. doi: 10.1029/2010JD015505.

[66] D. Kahaner, C. Moler, and S. Nash. Numerical Methods and Software. Prentice-Hall, Englewood Cliffs, NJ, 1989.

[67] F. Esposito, G. Grieco, G. Masiello, G. Pavese, R. Restieri, C. Serio, and V. Cuomo. Intercomparison of line-parameter spectroscopic databases using downwelling spectral radiance. Quart. J. R. Met. Soc., 133:191-202, 2007. doi: 10.1002/qj.131.

[68] L.S. Rothman, I.E. Gordon, R.J. Barber, H. Dothe, R.R. Gamache, A. Goldman, V.I. Perevalov, S.A. Tashkun, and J. Tennyson. HITEMP, the high-temperature molecular spectroscopic database. J. Quant. Spectrosc. \& Radiat. Transfer, 111(12-13):2139-2150, 2010. doi: 10.1016/j.jqsrt.2010.05.001.

[69] J. Tennyson, S.N. Yurchenko, A.F. Al-Refaie, E.J. Barton, K.L. Chubb, P.A. Coles, S. Diamantopoulou, M.N. Gorman, C. Hill, A.Z. Lam, L. Lodi, L.K. McKemmish, Y. Na, A. Owens, O.L. Polyansky, T. Rivlin, C. Sousa-Silva, D.S. Underwood, A. Yachmenev, and E. Zak. The ExoMol database: Molecular line lists for exoplanet and other hot atmospheres. J. Mol. Spectrosc., 327:73 - 94, 2016. doi: 10.1016/j.jms.2016.05.002. New Visions of Spectroscopic Databases, Volume II.

[70] K.P. Shine, A. Campargue, D. Mondelain, R.A. McPheat, I.V. Ptashnik, and D. Weidmann. The water vapour continuum in near-infrared windows - current understanding and prospects for its inclusion in spectroscopic databases. J. Mol. Spectrosc., 327:193 - 208, 2016. doi: 10.1016/j.jms.2016.04.011. New Visions of Spectroscopic Databases, Volume II.

[71] D.D. Turner, D.C. Tobin, S.A. Clough, P.D. Brown, R.G. Ellingson, E.J. Mlawer, R.O. Knuteson, H.E. Revercomb, T.R. Shippert, W.L. Smith, and M.W. Shephard. The QME AERI LBLRTM: A closure experiment for downwelling high spectral resolution infrared radiance. J. Atmos. Sci., 61(22):2657-2675, 2004. doi: 10.1175/JAS3300.1.

[72] L. Oreopoulos, E. Mlawer, J. Delamere, T. Shippert, J. Cole, B. Fomin, M. Iacono, Z. Jin, J. Li, J. Manners, P. Räisänen, F. Rose, Y. Zhang, M.J. Wilson, and W.B. Rossow. The continual intercomparison of radiation codes: Results from phase I. J. Geophys. Res., 117(D6):D06118, 2012. doi: 10.1029/ 2011JD016821.

[73] D.S. Amundsen, I. Baraffe, P. Tremblin, J. Manners, W. Hayek, N.J. Mayne, and D.M. Acreman. Accuracy tests of radiation schemes used in hot Jupiter global circulation models. Astron. \&6 Astrophys., 564:A59, 2014. doi: 10.1051/0004-6361/201323169.

[74] C. Richard, I.E. Gordon, L.S. Rothman, M. Abel, L. Frommhold, M. Gustafsson, J.-M. Hartmann, C. Hermans, W.J. Lafferty, G. Orton, K.M. Smith, and H. Tran. New section of the HITRAN database: Collision-induced absorption (CIA). J. Quant. Spectrosc. \& R Radiat. Transfer, 113(11): 1276-1285, 2012. doi: 10.1016/j.jqsrt.2011.11.004.

[75] M. López-Puertas and F.W. Taylor. Non-LTE Radiative Transfer in the Atmosphere. World Scientific Publishing, 2001. 